\title{
Microbial $\beta$-Glucosidases: Screening, Characterization, Cloning and Applications
}

\author{
Amer Ahmed,", Muhammad Aslam², Muhammad Ashraf ${ }^{2}$, Faiz ul-Hassan Nasim,, \\ Kashfa Batool ${ }^{2}$, Aasia Bibi ${ }^{2}$ \\ ${ }^{1}$ Department of Biochemistry and Biotechnology, Faculty of Science, The Islamia University of Bahawalpur, Pakistan \\ ${ }^{2}$ Department of Chemistry, Biochemistry Section, Faculty of Science, The Islamia University of Bahawalpur, Pakistan \\ *Corresponding author: aa.biotechiub@gmail.com, Faiznasim@hotmail.com
}

\begin{abstract}
Cellulose is the most abundant biomaterial in the biosphere and the major component of plant biomass. Cellulase is an enzymatic system required for conversion of renewable cellulose biomass into free sugar for subsequent use in different applications. Cellulase system mainly consists of three individual enzymes namely: endoglucanase, exoglucanase and $\beta$-glucosidases. $\beta$-Glucosidases are ubiquitous enzymes found in all living organisms with great biological significance. $\beta$-Glucosidases have also tremendous biotechnological applications such as biofuel production, beverage industry, food industry, cassava detoxification and oligosaccharides synthesis. Microbial $\beta$-glucosidases are preferred for industrial uses because of robust activity and novel properties exhibited by them. This review aims at describing the various biochemical methods used for screening and evaluating $\beta$-glucosidases activity from microbial sources. Subsequently, it generally highlights techniques used for purification of $\beta$-glucosidases. It then elaborates various biochemical and molecular properties of this valuable enzyme such as $\mathrm{pH}$ and temperature optima, glucose tolerance, substrate specificity, molecular weight, and multiplicity. Furthermore, it describes molecular cloning and expression of bacterial, fungal and metagenomic $\beta$-glucosidases. Finally, it highlights the potential biotechnological applications of $\beta$-glucosidases.
\end{abstract}

Keywords: lignocellulose biomass, cellulose, cellulase, $\beta$-glucosidase, biofuel, transglycosylation

Cite This Article: Amer Ahmed, Muhammad Aslam, Muhammad Ashraf, Faiz ul-Hassan Nasim, Kashfa Batool, and Aasia Bibi, "Microbial $\beta$-Glucosidases: Screening, Characterization, Cloning and Applications." Journal of Applied \& Environmental Microbiology, vol. 5, no. 2 (2017): 57-73. doi: 10.12691/jaem-5-2-2.

\section{Introduction}

Cellulose is the most abundant biomaterial in the biosphere and the major constituent of plant biomass representing 35-50\% of lignocellulose biomass [1]. Cellulase is an enzymatic system that converts renewable cellulose biomass into free sugar for the subsequent use in different application. Cellulase systems consist mainly of three enzymes namely: endoglucanase (1,4- $\beta$-D-glucan-4-glucanohydrolase; EC 3.2.1.4) and exoglucanase (1,4- $\beta$-D-glucan glucohydrolase; EC 3.2.1.74) and $\beta$-glucosidase ( $\beta$-D-glucoside glucohydrolase, EC 3.2.1.21) that synergistically degrade cellulose to glucose unit $[2,3,4]$. First, endoglucanase attacks the internal $\beta(1-4)$-linkages of cellulose chains releasing $\beta$-glucan chains of different length. Subsequently, exoglucanase acts on $\beta$-glucan chains from both reducing and nonreducing end releasing cellobiose, and short oligosaccharides. Finally, $\beta$-glucosidase degrades cellobiose and short oligosaccharides into glucose units $[5,6,7,8,9]$. In addition, $\beta$-glucosidase is diverse group of enzymes that catalyze liberation of nonreducing glucosyl terminal residue from different aryl-, alkyl- $\beta$-D-glucosides, $\beta$-linked disaccharides and short oligosaccharides [10,11]. $\beta$-Glucosidase is ubiquitous enzyme found in bacteria, fungi, plant, and animals including noncellulolytic organisms e.g., human $[12,13]$.

Glycoside hydrolases (GHs) is a widespread class of enzymes which catalyzes hydrolysis of glycosidic bonds between carbohydrates or a carbohydrate and a noncarbohydrate moiety. GHs comprise of enzymes GHs encompass 135 enzymes families based on nucleotide sequences identity and hydrophobic cluster analysis as in the last update of CaZy database [14]. $\beta$-Glucosidases are placed in $\mathrm{GH}$ family 1 and family 3 . $\beta$-Glucosidases belonging to $\mathrm{GH}$ family 1 are from bacteria, plants and mammals whereas those belonging to $\mathrm{GH}$ family 3 are from bacteria, fungi and plants [12,15].

$\beta$-Glucosidase plays fundamental roles in many biological processes in different organisms. For example, in human, at least five $\beta$-glucosidases have been identified which differ in their localization and substrates specificity [16]. Probably the most studied one is the lysosomal acid $\beta$-glucosidase (3.2.1.45) which hydrolyzes $\beta$-glucosyl linkage of glucosylceramide releasing ceramide and involved in Gaucher's disease pathogenesis [17]. In plants, $\beta$-glucosidases are involved in many physiological processes such as cell wall lignification [18], seed germination [19], phytohormones activation [20], indole alkaloids biosynthesis [21], cyanogenesis [22], and defense against biotic stresses by releasing of toxic 
compounds from their glycosides precursors [23]. In microorganism, $\beta$-glucosidases are localized as intracellular, extracellular and cell wall-associated enzyme and involved in degradation of cellulose and other carbohydrates for nutrients up-take and carbon recycling [24], cellulase gene induction [25], cell wall metabolism, host-pathogen interactions, and symbiotic association $[1,10,26]$.

Apart from physiological functions of $\beta$-glucosidases, these enzymes are used in many biotechnological applications such as food and flavors technology, paper recycling, cassava detoxification, and most importantly biofuel production by enhancing the rate of saccharification of lignocellulose biomass [27,28,29,30]. In addition, some $\beta$-glucosidase can catalyze synthetic activity under certain circumstances of high substrate or product concentration which shift reaction equilibrium toward synthesis and can therefore be utilized in the synthesis of oligosaccharides, aryl- and alkyl- $\beta$-glucosides $[12,16,31]$. These compounds are chemically stable, environmentally friendly, and have nonionic surfactants and biodegradable properties and have wide range of applications such as therapeutic agents, vaccines, cosmetics, diagnostics tools, and growth promoting agents for probiotic bacteria [32,33,34,35]. Researchers therefore are focusing on characterization of this valuable enzyme from microorganisms for their industrial applications rather than investigating physiological roles.

In this review, various biochemical methods for screening and measuring microbial $\beta$-glucosidase activity are described and general procedures for purification of $\beta$-glucosidases are highlighted. Subsequently, various biochemical and molecular properties such as $\mathrm{pH}$ and temperature optima, substrate specificity, glucose tolerance, transglycosylation, molecular weight, and multiplicity are elaborated. Furthermore, molecular cloning and expression of microbial $\beta$-glucosidase from bacteria, fungi and metagenome is discussed. Finally, potential industrial applications of $\beta$-glucosidase are highlighted.

\section{Screening for $\beta$-Glucosidases}

$\beta$-Glucosidase production by a microorganism grown in agar medium can be detected using esculin, 8-hydroxyquinoline- $\beta$-D-glucoside, cyclohexenoesculetin-
$\beta$-D-glucoside, or arbutin which upon hydrolysis by $\beta$-glucosidase gives rise to glucose and aglycone moiety: esculetin, hydroxyquinoline, cyclohexenoesculetin, and hydroquinone, respectively. Aglycone then chelates ferric ions supplemented to the growth medium (Figure 1), and forms dark brown complex against clear background [36,37]. The main disadvantage for using esculin is diffusion of esculetin-iron complex throughout medium making it difficult to distinguish $\beta$-glucosidase producing colonies from non-producers [38]. Hydroxyquinoline- $\beta$ $\mathrm{D}$-glucoside is known to be less diffusible in comparison to esculin, although it is toxic to gram positive bacteria [39]. Contrarily, cyclohexenoesculetin- $\beta$-glucoside allows the growth of gram-positive bacteria and does not suffer from the diffusion of the 8-hydroxyquinoline-iron complex throughout the plate [40]. The ferric salt may inhibit the growth of microorganisms and misinterpretation of the results may occur $[37,41]$.

Alternatively, MUG (methylumbelliferyl- $\beta$-D-glucoside) can be used to detect $\beta$-glucosidase activity on agar medium. MUG is an artificial glucoside that is hydrolyzed by $\beta$-glucosidase into methylumbelliferone and glucose. Methylumbelliferone is known to fluoresce when induced by UV light. Appearance of white zone around the colonies indicates that the microorganism is a $\beta$-glucosidase producer [42,43].

Indoxyl- $\beta$-D-glucosides such as 5-bromo-4-chloro-3indoxyl-, 5-bromo-6-chloro-3-indoxyl-, 6-chloro-3-indoxyl-, 5 -bromo-3 indoxyl- $\beta$-D-glucosides may be used to detect $\beta$-glucosidase which upon hydrolysis by the enzyme, the indoxyl derivative imparts specific color to the positive clone. For instance, 5-bromo-4-chloro-3-indoxyl forms blue color and 6-chloro-3-indoxyl forms rose color [44,45,46].

Perry et al utilized $\beta$-glucosides of alizarin (1,2 dihydroxyanthraquinone), 3,4-dihydroxyflavone and 3hydroxyflavone for the detection of $\beta$-glucosidase on plate and found that alizarin glucoside was highly sensitive for bacterial $\beta$-glucosidase, and 3,4-dihydroxyflavone and 3-hydroxyflavone were also sensitive for $\beta$-glucosidase from Enterococci and Listeria spp. [46]. Cellobiose was used for combinatorial screening and selection for $\beta$-glucosidase producers e.g., microorganism with $\beta$-glucosidase production capability can grow on media containing cellobiose as sole carbon source while those without $\beta$-glucosidase activity cannot grow [47].

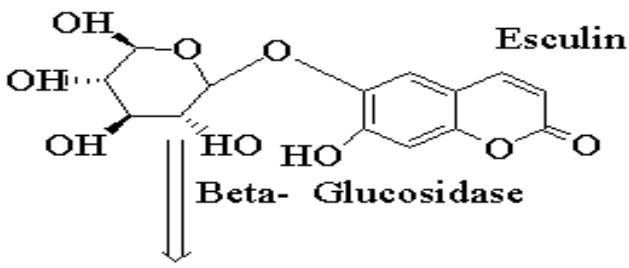<smiles>CC1(O)C(O)C(O)C(O)C1O</smiles><smiles></smiles>

In the presence of ferric ions<smiles>O=c1ccc2cc(O)c(O)cc2o1</smiles>

Glucose 


\section{Determination of $\beta$-Glucosidase Activity}

$\beta$-Glucosidase activity is most commonly measured using $p$-nitrophenyl- $\beta$-D-glucopyranoside (p-NPG) as substrate. $\beta$-Glucosidase hydrolyzes $\mathrm{p}-\mathrm{NPG}$ to glucose and $p$-nitrophenol. $p$-Nitrophenol, under alkaline conditions, turns yellow with optimal absorbance at $405 \mathrm{~nm}$ which can be measured using spectrophotometer. Generally $\beta$-glucosidase has a high affinity for this artificial substrate making it the most widely used substrate for measuring $\beta$-glucosidase activity [48,49,50,51].

$\beta$-Glucosidase activity can also be measured using cellobiose which is a natural disaccharide produced from cellulose by the action of endo- and exo-glucanase. Cellobiose is hydrolyzed by $\beta$-glucosidase into two glucose units. The liberated glucose is measured using glucose specific test such as glucose oxidase peroxidase (GOD/POD) kit, hexokinase kit, or High Pressure Liquid Chromatography (HPLC). Since cellobiose is the natural substrate for $\beta$-glucosidase, it is preferred to be utilized for evaluating its activity [52,53,54]. Other substrates such as salicin and arbutin have been used to evaluate the enzyme activity. The enzyme hydrolyzes salicin or arbutin to glucose and aglycone moiety either saligenen or hydroquinone, respectively, and the released glucose is measured using standard method such as DNS, or GOD/POD kit $[29,55,56]$. MUG may also be used for measuring $\beta$-glucosidase activity. The enzyme hydrolyzed MUG into $\beta$-methylumbelliferone and glucose. The amount of methylumbelliferone released by the enzymes is measured by fluorimeter for excitation and emission at $380 \mathrm{~nm}$ and $448 \mathrm{~nm}$, respectively [57,58].

Furthermore, cellotriose, cellotetraose, cellopentose and cellohexoses are used to evaluate $\beta$-glucosidase hydrolytic activity on short oligosaccharides [59]. Daidzin, and genistin are used to measure $\beta$-glucosidase capability to hydrolyze isoflavone glycosides $[60,61]$. Some $\beta$-glucosidase showed $\beta$-galactosidase side activity, and/ $\beta$-xylosidase side activity which can be measured using lactose, $p$-nitrophenol- $\beta$-D-galactoside, or $p$-nitrophenyl$\beta$-D-xylopyranoside, respectively $[62,63,64]$.

\section{Purification of $\beta$-Glucosidase}

Majority of industrial applications of $\beta$-glucosidase do not demand a homogeneous preparation of the enzyme. However, a highly pure enzyme is required for biochemical and molecular characterization, 3-D structure elucidation, and the structure-function relationships. $\beta$-Glucosidase therefore has been purified from different sources including fungi, bacteria and yeast and various strategies have been employed for $\beta$-glucosidase purifications $[65,66,67]$. The pre-purification step usually involves protein precipitation/fractionation from microbial culture using ammonium sulfate at 75\% [68], 80\% $[69,70,71,72]$, and $90 \%$ saturation [55,73]. Other workers have used ultrafiltration [66,74], acetone precipitation [75], and ethanol precipitation [76]. The next step usually involves the use of dialysis to remove ammonium sulfate traces and other impurities present in the culture medium
$[77,78]$. Further purification is done by chromatographic technique such as gel filtration chromatography, ions exchange chromatography, adsorption chromatography, hydrophobic interaction chromatography or HPLC $[68,71,79]$. For example, $\beta$-glucosidase has been purified up to 9.47 fold from culture supernatant of Tolypocladium cylindrosporum Syzx4 using ammonium sulfate (75\% saturation), dialysis, (DEAE)-cellulose column, and Sephadex G-100 column [68]. $\beta$-Glucosidase has also been purified from Aureobasidium pullulans using ammonium sulfate precipitation, CM Bio-Gel A agaraose, and sephacryl S-200 gel filtration chromatography up to 129 fold [80] and that from the human pathogenic fungus Sporothrix schenckii, was purified to homogeneity using hydroxyapatite (HAp) adsorption chromatography and Sephacryl S200-HR size exclusion chromatography [81]. Affinity chromatography methods such as Immobilized metal-affinity chromatography (IMAC) has been utilized for purification of recombinant enzymes containing short affinity tag of polyhistdine residues which show strong interactions with transition metal ions such as $\mathrm{Ni}^{2+}$ immobilized in matrix [82]. For instance, $\beta$-glucosidase from Anoxybacillus sp. DT3-1 [83], Exiguobacterium antarcticum B7 [84], and Thermoanaerobacterium thermosaccharolyticum DSM 571 [85] has been expressed in E. coli as fusion protein with six His tag at N-terminal and purified through Nickel-Nitrilotriacetic acid (Ni-NTA) chromatography [85,86,87]. Glutathione-S-Transferase (GST) affinity has been used for purification of recombinant $\beta$-glucosidase from Exiguobacterium oxidotolerans A011 expressed as fusion protein with GST which provides additional advantage by acting as a chaperone protein to facilitate protein folding, and provide a mean for their purification by immobilized glutathione column [88,89]. In general, $\beta$-glucosidase purification is quite troublesome, time-consuming and results in low purification yield. Therefore, novel purification strategies have yet to be adopted to reduce the number of purification steps and enhance the purification yield.

\section{Biochemical Properties of B-Glucosidase}

Subsequently after purification, $\beta$-glucosidases are characterized for their biochemical properties such as $\mathrm{pH}$ and temperature optima, substrate specificity, glucose tolerance, transglycosylation to determine potential biotechnological applications of the enzyme [90,91].

\section{1. pH and Temperature Optima}

$\beta$-Glucosidases differ in $\mathrm{pH}$ optima depending on their origin, and sources. Generally fungal $\beta$-glucosidase exhibits optimal activity at pH of 4.0-6.0 [59,69,86,92,93], nevertheless $\beta$-glucosidase with optimal $\mathrm{pH}$ of 2.4 has been reported from T. cylindrosporum Syzx4 [68] and that with optimal $\mathrm{pH}$ of 9 and 10 has been reported from Acremonium murorun LPSC 927 and Chaetomium globosum, respectively $[94,95]$. Bacterial $\beta$-glucosidase exhibits optimal activity at $\mathrm{pH}$ of 6-7 [44,87,96,97], although those with $\mathrm{pH}$ optima of 5,8 and 10 has been reported from Caldicellulosiruptor saccharolyticus [98], 
Bacillus halodurans [99], Klebsiella pneumoniae [100], respectively. Yeast $\beta$-glucosidase reported from $A$. pullulans and Candida peltata exhibited optimal activity at pH $5[80,101]$ and that from Kluyveromyces marxianus showed optimal activity at pH 5.5 [102]. The yeast-like Aureobasidium sp. was found to secrete highly acidophilic extracellular $\beta$-glucosidase with optimal activity at $\mathrm{pH} 2.5$ [103]. Metagenomic $\beta$-glucosidases exhibiting optimal activity at pH 7 [104], 6 [105,106], 6.5 [45,49,107], 5.5 [108,109], 4.0 [110], 8 and 10 [111,112] has been identified reflecting the great diversity of unculturable microbial world and its potential for production of industrially novel enzymes.

Similarly, $\beta$-glucosidases vary in optimum temperature with majority of the reported microbial $\beta$-glucosidases are thermophilic enzymes. For example, $\beta$-glucosidases exhibiting optimal activity at temperature of $50^{\circ} \mathrm{C}$ have been identified from Flammulina velutipes [113], Daldinia eschscholzii [65], Penicillium purpurogenum [114] and those with optimal activity at $60^{\circ} \mathrm{C}$ have been reported from Ceriporiopsis subvermispora [115], and Halothermothrix orenii [116]. $\beta$-Glucosidase with optimal activity at $70^{\circ} \mathrm{C}$ has been reported from A. niger KCCM 11239 [117], and T. aurantiacus IFO9748 [43]. Moreover, hyperthermophilic $\beta$-glucosidase has been identified from Pyrococcus furiosus with optimal temperature of $102-105^{\circ} \mathrm{C}$ [63] and $\beta$-glucosidase with optimal activity at $90^{\circ} \mathrm{C}$ has also been identified from hot spring metagenome and termite gut metagenome [105,107]. Mesophilic $\beta$-glucosidase has been reported from Neocallimastix patriciarum W5 [118], Neosartorya fischeri NRRL181 [86], and P. purpurogenum KJS506 [119] with optimal temperature of 40 , and $32^{\circ} \mathrm{C}$, respectively. Cold active $\beta$-glucosidase have been characterized from Paenibacillus sp. Strain C7 [87], and Shewanella sp. G5 [120], and E. oxidotolerans A011 [89]. This diversity in $\mathrm{pH}$ and temperature optima of microbial $\beta$-glucosidase reflects the wide distribution and fundamental roles played by this enzyme in all living organisms. Further studies are needed to determine the mechanisms by which this enzyme can work under high or low temperature and various $\mathrm{pH}$. Understanding of these mechanisms will eventually help in designing a better catalyst through protein engineering.

\subsection{Substrate Specificity}

$\beta$-Glucosidases are classified based on substrate specificity into three classes: 1) Cellobiase which catalyzes hydrolysis of cellobiose and short oligosaccharides, 2 ) Aryl- $\beta$-D-glucosidase which hydrolyzes only aryl- $\beta$-Dglucosides such as p-NPG and 3) Broad substrate specificity $\beta$-glucosidase which catalyze hydrolysis of wide range of substrates with $\beta(1-4), \beta(1-2), \beta(1-3), \alpha(1-4)$, $\alpha(1-2), \alpha(1-6)$ bonds etc. [10]. $\beta$-Glucosidase with broad substrate specificity has been identified from $F$. oxysporum [121], P. thermophile J18 [59], P. italicum [74], Penicillium simplicissimum [55], A. oryzae [122], Thermobifida fusca [123], and environmental DNA [49]. Cellobiase has been identified from Cellulomonas biazotea [124], and A. niger [52]. Aryl- $\beta$-D-glucosidase has been reported in $N$. fischeri NRRL181 [86] and A. oryzae [125]. Further studies, including molecular docking studies, are needed to determine interactions between amino acids residues with substrates which may provide an insight into these substrate specificities and allow better use of this multifunctional biocatalyst [126,127].

\subsection{Glucose Sensitivity and Tolerance}

Microbial $\beta$-glucosidases are inhibited by their own product i.e., glucose [128]. For instance, $\beta$-glucosidase reported in $F$. oxysporum was inhibited competitively by glucose with inhibitory constant $(\mathrm{Ki})$ value of $2.05 \mathrm{mM}$ [121], and $\beta$-glucosidase reported in $P$. italicum was inhibited non-competitively with $\mathrm{Ki}$ of $8.9 \mathrm{mM}$ [74]. Other $\beta$-glucosidases characterized from Termitomyces cyleaputs [129], Fomitopsis palustris [66], Monascus purpureus NRRL1992 [93], D. eschscholzii [65], Gongronella butleri [130], and Leuoconstic Mesentenoide [29] were also sensitive to glucose with $\mathrm{Ki}$ value range of $0.35-14.3 \mathrm{mM}$.

$\beta$-Glucosidase with an excellent glucose tolerance has also been identified from different species such as $C$. peltata [131], A. unguis NII-08123 [132], A. niger CCRC 31494 [133], A. oryzae [122] and Penicillium funiculosum NCL1 [134] with Ki value of 1.4, 0.8, 0.5 and $1.36 \mathrm{mM}$, respectively. Moreover, $\beta$-glucosidases belonging to $\mathrm{GH}$ family 1 was found to be stimulated by lower glucose concentration and be tolerant to higher concentration. For examples, $\beta$-glucosidase from Microbispora bispora was activated in the presence of $300 \mathrm{mM}$ glucose [135] and that reported in T. thermosaccharolyticum DSM 571 and T. aotearoense was activated by glucose concentration $<200$ and $250 \mathrm{mM}$, respectively [85,136]. Similarly, recombinant GH $1 \beta$-glucosidase from Exiguobacterium antarcticum B7 [84], Fervidobacterium islandicum [137], Thermotoga thermarum DSM 5069T [138] and Anoxybacillus DT3-1 [83] was tolerant to high glucose concentration e.g., $\mathrm{Ki}$ of $0.54-15 \mathrm{M}$. On the other hand, glucose tolerant $\beta$-glucosidase with $\mathrm{Ki}$ of 1.0-4.8 $\mathrm{M}$ has been identified from different environments using metagenomic approaches [45,53,106,111,139,140].

Glucose inhibition of $\beta$-glucosidases is the major obstacle in bioconversion of biomass and biofuel production. Understanding the mechanism of glucose inhibition/tolerance is of crucial importance for biofuel production. Recently, replacement of two amino acids (Leu 167 and Pro 172) at the entrance of the active site of intracellular $\beta$-glucosidase of $T$. reesei (Bgl II) with Trp and Leu, respectively, significantly enhanced glucose tolerance e.g., $\mathrm{Ki}$ of $650 \mathrm{mM}$ [141]. Further studies for identification of new $\beta$-glucosidase with high glucose tolerance are required. Bioinformatics analysis may be used for identification of amino acid residues playing central role in glucose tolerance and in silico mutagenesis and docking studies can be used to modulate this enzyme for better performance as preliminary experiment before protein engineering is employed.

\subsection{Transglycosylation Activity}

Transglycosylation is transfer of sugar moiety from one compound (donor) to another compound (accepter). Transglycosylation fundamentally is an important reaction for production of many compounds such as aryl/alkyl-, 
poly glycosides, and synthetic oligosaccharides e.g., galacto-oligosaccharides and gentio-oligosaccharides [142]. Both glycosyltransferases and glycosidases can be utilized for catalyzing transglycosylation. Glycosyltransferases require an input of energy in form of nucleotide triphosphate and have very narrow substrate specificity. Glycosidases, on the other hand, do not require an input of energy, use cheap donor substrates, and has relaxed substrate specificity for acceptors and are wide spread enzymes. Although glycosidases suffer from limitations such as product hydrolysis and low transglycosylation activity $[143,144]$. In this context, some $\beta$-glucosidase can catalyze transglycosylation under high substrate or product concentration [31]. In this reaction, the nucleophilic residue at active site attacks the glycosidic bond of nonreducing glucosyl terminal unit forming glucosyl-enzyme intermediate. The leaving group e.g. aglycone subtracts a proton from general acid/base residue before leaving the active site. Another acceptor molecule competes with the water molecule to attack enzymeglucosyl intermediate displacing nucleophilic residue and catalytic acid/base accepts a proton from the hydroxyl group of the acceptor [31,145,146]. Accepter molecule can be glucose, cellobiose, cellotriose, methanol, ethanol or propanol forming cellobiose, cellotriose, cellotetraose, methyl, ethyl, and propyl- $\beta$-glucosides, respectively [147].

$\beta$-Glucosidase with an excellent transglycosylation activity has been identified from different sources such as P. thermophile [59], Myceliophthora thermophile [79], T. aurantiacus [147], F. oxysporum [121], Melanocarpus sp. MTCC 3922 [148], T. clypeatus [149], and compost microbial metagenome [108]. Understanding mechanism of transglycosylation and identification of amino acid residues playing central role in transglycosylation reactions hold a great importance in protein engineering for the development of $\beta$-glucosidase with high transglycosylation reaction. Replacement of asparagine residue to phenylalanine at position 220 of $\mathrm{GH} 1$ $\beta$-glucosidase of Thermotoga neapolitana resulted in 7-fold increase in the transglycosylation reactions [142].

Researchers are therefore focusing on identification of new $\beta$-glucosidases with strong transglycosylation activity. Further mechanistic studies may give an insight to the mechanism of transglycosylation and amino acid residues playing central role in this valuable reaction. Understanding transglycosylation mechanism will eventually help in designing enzymes with efficient transglycosylation activity for better glycoside and oligosaccharides synthesis.

\subsection{Organic Solvents and Metal Ions Effect}

For $\beta$-glucosidase to be used in biofuel production and beverage industries, it has to be tolerant to ethanol and methanol, butanol, acetic acids, the main fermentation products $[122,130]$. Furthermore synthetic reactions e.g., transglycosylation uses organic solvents to shift reaction equilibrium from hydrolysis to synthesis [150]. As a result, organic solvent-tolerant $\beta$-glucosidases are of great significance to these biotechnological applications. A number of $\beta$-glucosidase has been identified with tolerance to organic solvent. $\beta$-Glucosidase activity from A. niger was increased by $30 \%$ and $80 \%$ in the presence of $30 \%$ ethanol and methanol, respectively [73]. Thermostable $\beta$-glucosidase from $F$. islandicum was activated in the presence of $99 \%$ of hexadecane, n-hexane, heptane, isooctane, amylalcohol, n-decyl alcohol by 2, 5, 28, 10, 28 and 23\%, respectively, and was slightly inhibited in the presence of $99 \%$ of tert-butanol, ethanol, acetonitrile, isopropanol, pyridine, DMSO, acetone, dimethylformamide, and methanol [137]. High glucosetolerant $\beta$-glucosidase from $A$. oryzae was stimulated by $30 \%$ in the presence of $15 \%$ ethanol [122]. Methanol and ethanol $(50 \%)$ stimulated $\beta$-glucosidase from $F$. velutipes by 5 and 23\%, respectively [113]. $\beta$-Glucosidase activity of Melanocarpus sp. was enhanced by 1.5 fold in the presence of $70 \%$ methanol and ethanol whereas in the presence of $70 \%$ propanol it retained complete activity [148]. $\beta$-Glucosidase of $F$. oxysporum was stimulated by $0.5 \mathrm{M}$ butanol (2.2-fold) and $1 \mathrm{M}$ methanol (1.4 Fold) [121]. $\beta$-Glucosidase from $M$. thermophile was highly activated by low molecular weight alcohol e.g., 1.4 fold increase in activity was achieved in the presence of $10 \%$ propanol, $15 \%$ ethanol and 20\% methanol [79]. G. butleri produced a $\beta$-glucosidase which was activated in the presence of 5\% ethanol up to 40\% [130] and Rhizomucor miehei NRRL 5282 secreted $\beta$-glucosidase which was activated by $40 \%$ in the presence of $15 \%$ ethanol.
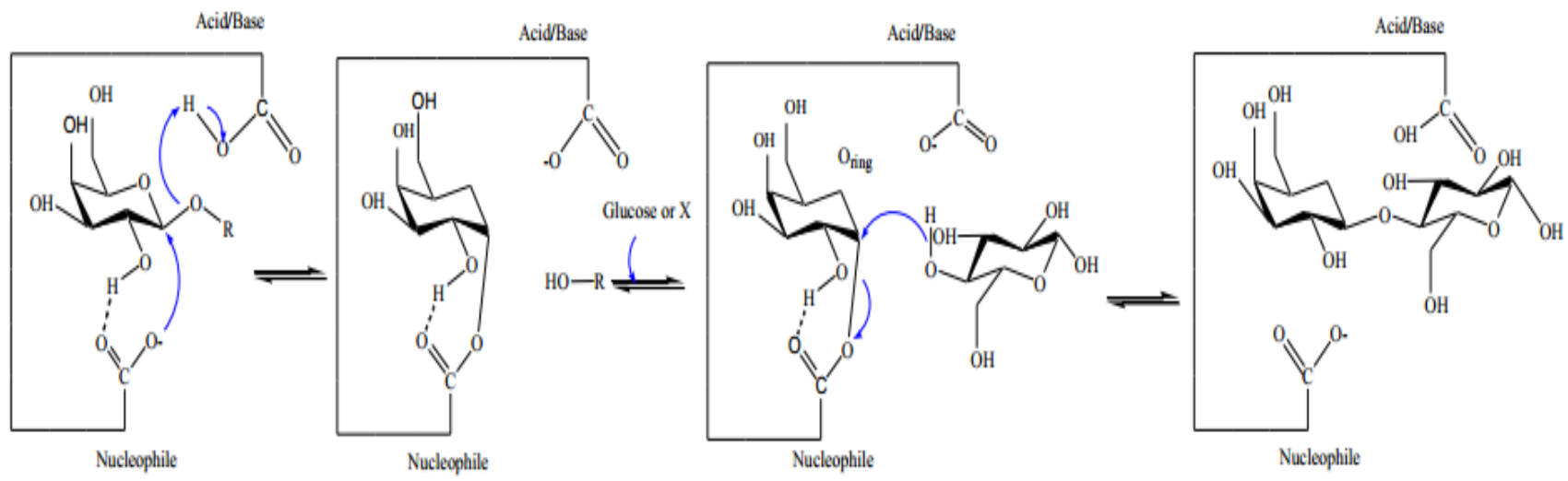

Figure 2. Transglycosylation mechanism of $\beta$-glucosidase 
$\beta$-Glucosidase is a metal-independent enzyme that it does not require metal ion for its activity. Nevertheless, it has been demonstrated that $\beta$-glucosidase is deferentially affected by different metal ions [55,72,151,152]. For instance, a cold-adapted $\beta$-glucosidase from $E$. antarcticum B7 was activated by $\mathrm{Mg}^{2+}, \mathrm{Na}^{+}, \mathrm{K}^{+}$, and $\mathrm{Li}^{3+}$, and inhibited by $\mathrm{Co}^{2+}, \mathrm{Mn}^{2+}, \mathrm{Ca}^{2+}, \mathrm{Zn}^{2+}, \mathrm{Cu}^{2+}, \mathrm{Fe}^{3+}[84]$ whereas the recombinant $\beta$-glucosidase from $M$. thermophile was highly activated by $\mathrm{Zn}^{2+}, \mathrm{Cu}^{2+}, \mathrm{Mg}^{2+}$, and $\mathrm{Mn}^{2+}$ and inhibited by $\mathrm{Ca}^{2+}$ and $\mathrm{Co}^{2+}$ [79]. $\beta$-Glucosidase from Ceriporiopsis subvermispora was stimulated by $\mathrm{Mn}^{2+}, \mathrm{Fe}^{3+}$ and inhibited by $\mathrm{Cu}^{2+}, \mathrm{Zn}^{2+}, \mathrm{Mg}^{2+}$, and $\mathrm{K}^{+}$. $\beta$-Glucosidase from Pseudonocardia sp. Gsoil 1536 was stimulated by $\mathrm{Ca}^{2+}, \mathrm{Mg}^{2+}, \mathrm{Mn}^{2+}, \mathrm{K}^{2+}, \mathrm{Co}^{2+}, \mathrm{Na}^{+}$, and $\mathrm{Zn}^{2+}$ and completely inhibited by $\mathrm{Hg}^{2+}$ [44]. The exact mechanism by which these metal ions exert their effect on $\beta$-glucosidase either stimulation or inhibition is not understood. Nevertheless, it is generally known that the inactivation caused by $\mathrm{Hg}^{2+}$ indicates that thiol groups are required for function of the enzyme or for maintaining 3D structure of the protein [122]. Inhibition by $\mathrm{Cu}^{2+}, \mathrm{Co}^{2+}$ and $\mathrm{Zn}^{2+}$ suggests that basic (Arg, Lys, His) and acidic (Asp, Glu) amino acids may have important roles in the active site and stimulation of enzyme activity by the cations $\mathrm{Ca}^{2+}$, $\mathrm{K}^{+}, \mathrm{Co}^{2+}, \mathrm{Mn}^{2+}, \mathrm{Mg}^{2+}$ and $\mathrm{Na}^{+}$suggest that it may enhance the structural stability of the enzyme $[153,154]$.

\section{Molecular Characterization of $\beta$-Glucosidase}

Molecular characterization of enzymes is of great importance for exploring the potential biotechnological applications. Microbial $\beta$-glucosidases from different sources have been characterized from different sources for molecular properties [155].

\subsection{Molecular Weight}

$\beta$-Glucosidase varies in their molecular weight depending on number of amino acids and posttranslational modifications e.g., glycosylation. Generally $\beta$-glucosidases belonging to GH 1 have 400-550 amino acids in length with molecular weight ranges $40-60 \mathrm{kDa}$ $[64,156]$. Similarly, $\beta$-glucosidases belonging to $\mathrm{GH}$ family 3 contain 600-900 amino acids in length with molecular weight of 65-90 kDa per subunit, but because these group of enzymes are usually glycosylated, their molecular mass ranges $110-130 \mathrm{kDa}[44,157,158]$. $\beta$-Glucosidase varies in quaternary structure arrangement, for example, monomeric [159], dimeric [160], trimeric [147], tetrameric [151] enzymes have been reported. Native molecular weight therefore may be higher than identified by SDS-PAGE [161]. For instance, $\beta$ glucosidase reported from $P$. italicum had a native molecular weight of $354 \mathrm{kDa}$ as determined by gel filtrations and $88.5 \mathrm{kDa}$ as shown by SDS-PAGE suggesting that the native protein is a tetramer [74]. Similarly, the predicted molecular weight from amino acids sequences may differ from that determined by SDS-PAGE due to post-translational modification [162]. For instance, Chen et al reported $\beta$-glucosidase from $P$. decumben with predicted molecular weight of $96 \mathrm{kDa}$ and
$120 \mathrm{kDa}$ as determined by SDS-PAGE [163]. Two short alkaline $\beta$-glucosidases have been identified with 172 and 151 amino acids length and molecular weight of 27 and 26 $\mathrm{kDa}$, respectively [112]. A novel intracellular $\beta$ glucosidase from $T$. clypeatus with molecular mass of $6.68 \mathrm{kDa}$ and $116 \mathrm{kDa}$ as determined by MALDI-TOF and SDS-PAGE, respectively, indicating that it is present in aggregate form [149].

\subsection{Zymography}

For the detection of $\beta$-glucosidase activity on gel matrix, number of substrates has been used by different researchers to visualize $\beta$-glucosidase on gel matrix $[42,164,165]$. For examples, Kwon et al used esculin to visualize $\beta$-glucosidase activity on Native-PAGE. The gel was removed and soaked in solution containing $0.1 \%$ esculin and $0.03 \%$ ferric ammonium citrate until a dark band appeared on the gel indicating the presence of $\beta$ glucosidase activity $[139,166,167]$. $P$-PNG has also been utilized to detect $\beta$-glucosidase activity on PAGE. The protein sample with $\beta$-glucosidase activity is mixed with SDS-PAGE buffer and loaded into native PAGE system and run for specific period of time. The gel is then incubated in p-NPG solution, and then immersed $\mathrm{Na}_{2} \mathrm{CO}_{3}$ solution till yellow bands appeared indicating the presence of $\beta$-glucosidase at that site [42,164]. MUG is another powerful substrate for visualization of $\beta$-glucosidase on gel matrix. Protein sample with $\beta$-glucosidase activity is loaded on native PAGE and run for specific period of time. After which the gel is incubated in buffered MUG solution and then visualized under UV light using UV transilluminator. Appearance of fluorescent bands indicates the liberation of methylumbelliferone due to enzyme activity [93,154,168,169].

\subsection{Multiplicity of $\beta$-Glucosidase}

Isoenzymes are the enzymes which catalyze the same biochemical reaction but differ in their composition e.g., amino acid sequences. $\beta$-Glucosidase multiplicity has been reported in number of filamentous fungi and yeast. For instances, multiplicity of $\beta$-glucosidase has been demonstrated in $T$. reesei $[170,171]$. A. niger NII-08121/MTCC 7956 was found to express four $\beta$-glucosidase isoforms when grown on lactose or cellulose, whilst 2 isoforms were expressed on wheat bran or rice straw as carbon source [172]. Sonia et al isolated thirteen thermophilic and thermotolerant fungi from composting soil and found that twenty-eight $\beta$-glucosidase isoforms were expressed when corn cob was used as carbon source. For instance, A. caespitosus produces four isoforms, Chaetomium thermophilum produced three isoforms, and Absidia corymbifera expressed 2 isoforms [173]. A. tubingensis CBS 643.92 expressed four $\beta$-glucosidases (I-IV) with MW of 131, 126, 54 and $54 \mathrm{kDa}$ and an isoelectric points of $4.2,3.9,3.7$ and 3.6 , respectively [174]. A. oryzae secreted two isoforms when cultured on media containing various carbon source: major form with MW of $130 \mathrm{kDa}$ and low glucose tolerance, and minor form with $\mathrm{MW}$ of $43 \mathrm{kDa}$ and high glucose tolerance [122]. C. subvermispora expressed two distinct $\beta$ glucosidase isoforms with molecular weight of 110 and 53 
kDA when grown under Solid State Fermentation (SSF) on $P$. taeda wood chips [115]. The thermotolerant $A$. terreus AN1 strain secreted four distinct $\beta$-glucosidase isoforms when corn cob was used as a carbon source. Three isoforms designated as $\beta$ GI, $\beta$ GII \& $\beta$ GIII had a MW of 29, 43, and $98 \mathrm{KDa}$, and isoelectric point of 2.8, 3.7, and 3.0, respectively [165]. Similarly, the filamentous fungus, Penicillium funiculosum NCL1, expressed multiple isoforms of $\beta$-glucosidase when cultivated on media containing different carbon source e.g., four isoforms on wheat bran, two isoforms on sugarcane bagasse, and one isoform on lactose containing media whereas no isoform was expressed on salicin containing media as sole carbon source [175]. A. unguis NII-08123 expressed five different $\beta$-glucosidase isoforms among which one novel isoform was highly glucose-tolerant with MW of $10 \mathrm{kDa}$ [132]. In bacteria, Bacillus subtilis strain PS secreted three isoforms $\beta$-glucosidase with molecular mass of $193 \mathrm{kDa}, 64 \mathrm{kDa}$ and $42 \mathrm{kDa}$, when it was grown on glucose containing media [176].

Thus, the major factor influencing the expression of these isoforms is the carbon sources e.g., $\beta$-glucosidase are usually inducible enzymes synthesized by microorganisms in response to certain metabolites present the culture medium. These metabolites usually are low molecular weight carbohydrates incorporated in the culture medium or synthesized by constitutively expressed enzymes secreted to the medium [24]. Other factors which may influence the expression of these isoforms are their production method either Solid Sate Fermentation (SSF) or Submerged Fermentation $(\mathrm{SmF})$, and fermentation conditions such as aeration, $\mathrm{pH}$, temperature and nitrogen sources [177]. The mechanism through which these isoforms are generated is not well understood, although probably these isoform are produced by gene multiplicity, alternative splicing, and post-translational modifications [24]. Further studies are needed to pursue the exact mechanism through which the expression of different isoforms are being regulated is of crucial significance for industries e.g., in the designing of culture parameters for production of the desired isoforms. List of $\beta$-glucosidase produced from different fungi, yeast and bacteria along with their biochemical and molecular properties are given in Table 1.

Table 1. List of the Characterized Fungal, Yeast, and Bacterial $\beta$-Glucosidases

\begin{tabular}{|c|c|c|c|c|c|c|c|}
\hline Source organism & GH Family & No. of subunits & MW(kDa) & pH Opt. & Temp. Opt & Substrate specificity & Reference \\
\hline P. thermophile & NR & Homodimer & 197 & 6.2 & 75 & Broad specificity & [59] \\
\hline A. niger Au0847 & $\mathrm{CH} 3$ & Heterodimer & $110+120$ & 4.6 & 65 & Broad specificity & [69] \\
\hline P. italicum & NR & Homotetramer & $88.5 / 354$ & 4.5 & 60 & Broad specificity & [74] \\
\hline P. simplicissimum & NR & Monomer & 126 & 4.8 & 60 & Broad specificity & {$[55]$} \\
\hline A. awamori MIBA335 & CH 3 & monomer & 120 & 4.5 & 55 & Broad specificity & [227] \\
\hline A. oryzae & NR & monomer & 43 & 5 & 50 & Broad specificity & [122] \\
\hline Fomitopsis palustri & $\mathrm{CH} 3$ & Monomer & 135 & 4.5 & 70 & Cellobiose + p-NPG & {$[66]$} \\
\hline T. aurantiacus & $\mathrm{CH} 3$ & Homotrimer & $120 / 350$ & 4.5 & 80 & Broad specificity & [147] \\
\hline T. lanuginosus-SSBP & $\mathrm{CH} 3$ & Homodimer & 200 & 6.0 & 65 & Broad specificity & {$[70]$} \\
\hline Melanocarpus sp. & NR & Monomer & 92 & 6.0 & 60 & Broad specificity & {$[148]$} \\
\hline A. fumigatus Z5 & $\mathrm{CH} 3$ & Monomer & 91.47 & 6.0 & 60 & Broad specificity & {$[72]$} \\
\hline Phoma sp. & $\mathrm{CH} 3$ & Tetramer & $110 / 440$ & 4.5 & 60 & Broad specificity & {$[151]$} \\
\hline D. eschscholzii & $\mathrm{CH} 3$ & Monomer & 64.2 & 5.0 & 50 & Broad specificity & {$[65]$} \\
\hline P. purpurogenum & $\mathrm{CH} 3$ & Monomer & 89.6 & 4 & 32 & Broad specificity & {$[119]$} \\
\hline F. velutipes & NR & Monomer & 75 & $5.0-6.0$ & 50 & $\mathrm{p}-\mathrm{NPG}$ & {$[113]$} \\
\hline P. piceum & $\mathrm{CH} 3$ & Monomer & 92 & 5.0 & 60 & Broad specificity & {$[228]$} \\
\hline P. decumbens & $\mathrm{CH} 3$ & Monomer & 120 & $4.5-5.0$ & $65-70$ & Broad specificity & {$[163]$} \\
\hline Penicillium citrinum & $\mathrm{CH} 3$ & Monomer & 72 & 5.0 & 70 & Broad specificity & [229] \\
\hline A. niger NII-08121 & $\mathrm{CH} 3$ & Monomer & 120 & 4.8 & 70 & Broad specificity & {$[172]$} \\
\hline Paecilomyces Bainier & $\mathrm{CH} 3$ & Monomer & 115 & 3.5 & 60 & Broad specificity & {$[230]$} \\
\hline Termitomyces clypeatus & NR & Aggregate & $6.68 / 116$ & 5.0 & 45 & Broad specificity & {$[149]$} \\
\hline A. terreus EMOO 6-4 & $\mathrm{CH} 3$ & Monomer & 120 & 5.5 & 35 & $\mathrm{p}-\mathrm{NPG}$ & {$[231]$} \\
\hline Agaricus bisporus & NR & Heterdimer & $62+46$ & 4.0 & 55 & Broad specificity & {$[232]$} \\
\hline T. citrinoviride & $\mathrm{CH} 3$ & Monomer & 110 & 4.0 & 50 & Broad specificity & {$[233]$} \\
\hline Sporothrix schenckii, & $\mathrm{CH} 3$ & Dimer & $97 / 204.9$ & 5.5 & 45 & Broad specificity & {$[160]$} \\
\hline Rhizomucor miehei & $\mathrm{CH} 3$ & monomer & 82.3 & 5.0 & 70 & Broad specificity & {$[153]$} \\
\hline Stachybotrys microbispora & $\mathrm{CH} 3$ & Monomer & 78 & 6.0 & 50 & Broad specificity & {$[234]$} \\
\hline P. funiculosum & $\mathrm{CH} 3$ & Monomer & 120 & 5.0 & 60 & Broad specificity & {$[175]$} \\
\hline A. niger KCCM 11239 & $\mathrm{CH} 3$ & Monomer & 123 & 4.0 & 70 & Broad specificity & {$[117]$} \\
\hline F. proliferatum ECU2042 & $\mathrm{CH} 3$ & Monomer & 78.7 & 5.0 & 50 & Broad specificity & {$[235]$} \\
\hline Humicola grisea & NR & Monomer & 57 & 6.0 & 50 & Broad specificity & {$[236]$} \\
\hline C. peltata & NR & Monomer & 43 & 5.0 & 50 & Broad specificity & [131] \\
\hline Penicillium janthinellum & NR & Monomer & 120 & 4.5 & 65 & Broad specificity & {$[237]$} \\
\hline
\end{tabular}

$\mathrm{NR}=$ not reported, $\mathrm{MW}=$ molecular weight, $\mathrm{kDa}=$ kilodalton, $\mathrm{pH}$ opt.= pH optima, Temp. opt.= temperature optima. 
Table 2. List of Fungal, Bacterial and Metagenomic Recombinant $\beta$-Glucosidases

\begin{tabular}{|c|c|c|c|c|c|c|c|c|}
\hline \begin{tabular}{|l|} 
Origin of BGL gene \\
Fungal species \\
\end{tabular} & $\begin{array}{c}\text { Host for } \\
\text { expression }\end{array}$ & $\begin{array}{c}\text { GH } \\
\text { Family }\end{array}$ & $\begin{array}{l}\text { No. of } \\
\text { a.a }\end{array}$ & $\begin{array}{l}\text { MW } \\
\text { kDa }\end{array}$ & $\begin{array}{c}\text { pH } \\
\text { Optima }\end{array}$ & $\begin{array}{c}\text { Temp } \\
\text { optima }\end{array}$ & Potential applications & Reference \\
\hline A. niger & P. pastoris. & $\mathrm{CH} 3$ & 860 & 116 & 4.0 & 60 & Biofuel production & {$[92]$} \\
\hline A. fumigatus Z5 & P. pastoris & $\mathrm{CH} 3$ & 844 & 130 & 6.0 & 60 & Biofuel production & [238] \\
\hline Periconia sp. & T. reesei & $\mathrm{CH} 3$ & 867 & 130 & 5.0 & 70 & Biofuel production & {$[184]$} \\
\hline Volvariella volvacea & E. Coli & CH3 & NR & 97 & 6.4 & 50 & Biofuel production & [239] \\
\hline Neosartorya fischeri & E. coli & CH 1 & 488 & $56 / 480$ & 6.0 & 40 & Food Technology & {$[86]$} \\
\hline Neosartorya fischeri P1 & P. pastoris & $\mathrm{CH} 3$ & 739 & 80 & 5.0 & 80 & Isoflavone hydrolysis & {$[155]$} \\
\hline T. emersonii & T. reesei & $\mathrm{CH} 3$ & 857 & 130 & 5.0 & 71.5 & Biofuel production & {$[187]$} \\
\hline T. reesei & T. reesei & $\mathrm{CH} 3$ & 744 & 75 & 4.6 & 70 & Biofuel production & {$[190]$} \\
\hline M. thermophila, & P. pastoris. & $\mathrm{CH} 3$ & 733 & 90 & 5.0 & 70 & Biofuel production & {$[79]$} \\
\hline A. saccharolyticus & T. reesei & $\mathrm{CH} 3$ & 860 & 130 & 4.2 & $50-60$ & Biofuel production & [240] \\
\hline T. reesei & P. pastoris & $\mathrm{CH} 3$ & 744 & 76 & 5.0 & 70 & Biofuel production & {$[180]$} \\
\hline Aspergillus aculeatus & T. reesei & $\mathrm{CH} 3$ & 841 & 130 & 5.5 & 65 & Biofuel production & [191] \\
\hline T. aurantiacus & P. pastoris & $\mathrm{CH} 3$ & 861 & 130 & 5.0 & 70 & Biofuel production & {$[43]$} \\
\hline P. brasilianum & A.oryzae & $\mathrm{CH} 3$ & 842 & 115 & 4.8 & 70 & Biofuel production & [183] \\
\hline P. funiculosum NCL1 & P. pastoris & $\mathrm{CH} 3$ & 857 & 130 & 5.0 & 60 & Bioethanol production & [134] \\
\hline N. patriciarum W5 & P. pastoris & $\mathrm{CH} 3$ & 776 & 85.1 & $5.0-6.0$ & 40 & Biofuel production & {$[118]$} \\
\hline Periconia sp. & P. pastoris & $\mathrm{CH} 3$ & 866 & 130 & 5.0 & 70 & Biofuel production & [241] \\
\hline N. fischeri NRRL181 & E. coli. & $\mathrm{CH} 1$ & 529 & 60 & 6.0 & 40 & Food technology & {$[242]$} \\
\hline \multicolumn{9}{|l|}{ Bacterial species } \\
\hline C. saccharolyticus & E. coli & CH1 & 453 & 54 & 5.5 & 70 & Biofuel production & {$[98]$} \\
\hline Exiguobacterium sp. & E. coli & $\mathrm{CH} 1$ & 450 & 52 & 7 & 45 & Oligosaccharide synthesis & [199] \\
\hline $\begin{array}{l}\text { Exiguobacterium } \\
\text { oxidotolerans A011 }\end{array}$ & E. coli & $\mathrm{CH} 1$ & 448 & 51.6 & 7 & 35 & Food technology & {$[89]$} \\
\hline Halothermothrix orenii, & E. coli & $\mathrm{CH} 1$ & 451 & 53 & 6.0 & $65-75$ & $\begin{array}{l}\text { Galactooligosaccharide } \\
\text { synthesis }\end{array}$ & [116] \\
\hline Weissella cibaria 37 & E. coli & $\mathrm{CH} 1$ & 415 & 50 & 5.5 & 45 & Biofuel production & {$[243]$} \\
\hline Sphingomonas sp. 2F2 & E. coli & $\mathrm{CH} 1$ & 447 & 49.3 & 5.0 & 37 & Ginsenoside transformation & [244] \\
\hline Pseudonocardia sp. & E. coli & $\mathrm{CH} 3$ & 743 & 79 & 7.0 & 37 & Ginsenoside transformation & {$[44]$} \\
\hline Bacteroides uniformis & E. coli & $\mathrm{CH} 3$ & 740 & 81.5 & 6.0 & 40 & $\begin{array}{l}\text { Secoisolariciresinol } \\
\text { diglucoside hydrolysis }\end{array}$ & {$[245]$} \\
\hline F. islandicum & E. coli & $\mathrm{CH} 1$ & 459 & 53 & $6.0-7.0$ & 90 & Biofuel production & {$[137]$} \\
\hline Thermobifida fusca, & E. coli & $\mathrm{CH} 1$ & 485 & 53.4 & 7.0 & 50 & Biofuel production & {$[123]$} \\
\hline T. thermosaccharolyticum & E. coli. & $\mathrm{CH} 1$ & 443 & 52 & 6.4 & 70 & Biofuel production & [246] \\
\hline Bacillus licheniformis & E. coli. & CH1 & 471 & 53 & 6.0 & 50 & Biofuel production & [200] \\
\hline $\begin{array}{l}\text { Thermoanaerobacter } \\
\text { brockii }\end{array}$ & E. coli & $\mathrm{CH} 1$ & 450 & 52 & 5.5 & 75 & Biofuel production & [247] \\
\hline Bacillus halodurans & E. coli & $\mathrm{CH} 1$ & 447 & 51.6 & 8.0 & 45 & Biofuel production & [99] \\
\hline Bacillus licheniformis & E. coli & $\mathrm{CH} 1$ & 469 & 53.4 & 6.0 & 47 & Biofuel production & [248] \\
\hline Anoxybacillus sp. DT3-1 & E. coli & $\mathrm{CH} 1$ & 453 & 53 & 8.5 & 70 & Biofuel production & {$[83]$} \\
\hline E. antarcticum B7 & E. coli & $\mathrm{CH} 1$ & 448 & 52 & 7.0 & 30 & $\begin{array}{l}\text { Biofuel production \& Food } \\
\text { Technology }\end{array}$ & {$[84]$} \\
\hline T. aotearoense & E. coli & CH 1 & 446 & 52.6 & 6.0 & 60 & Bioethanol production & {$[136]$} \\
\hline Thermotoga thermarum & E. coli & $\mathrm{CH} 1$ & 490 & 55 & 4.8 & 90 & Ginsenoside hydrolysis & {$[138]$} \\
\hline Bacillus halodurans & E. coli & GH 1 & 453 & 52.5 & 7.0 & 50 & Biofuel production & [249] \\
\hline Streptomycete sp. & E. coli & CH 1 & 456 & 51 & 6.0 & 45 & Biofuel production & {$[152]$} \\
\hline \multicolumn{9}{|l|}{ Metagenomics BGL } \\
\hline Marine metagenomic & E. coli & CH 1 & 442 & 50 & 7.5 & 45 & Food Technology & [64] \\
\hline Soil metagenome & E. coli & $\mathrm{CH} 1$ & 469 & 51.6 & 6.0 & 50 & Bioethanol production & {$[106]$} \\
\hline $\begin{array}{l}\text { Kusaya gravy } \\
\text { metagenome }\end{array}$ & E. coli & $\mathrm{CH} 1$ & 452 & 54 & $5.0-6.5$ & 45 & $\begin{array}{l}\text { Biofuel production } \\
\text { Food technology } \\
\end{array}$ & [250] \\
\hline Hot spring metagenome & E. coli & $\mathrm{CH} 1$ & 495 & 57.2 & 6.5 & 90 & Biofuel production & {$[107]$} \\
\hline Marine metagenomic & E. coli & $\mathrm{CH} 1$ & 442 & 51 & 6.5 & 40 & Biofuel production & [139] \\
\hline $\begin{array}{l}\text { Agricultural soil } \\
\text { metagenome }\end{array}$ & E. coli & $\begin{array}{l}\mathrm{CH} 3 \\
\mathrm{CH} 1 \\
\end{array}$ & $\begin{array}{l}762 \\
475 \\
\end{array}$ & $\begin{array}{l}80.9 \\
54.8\end{array}$ & $\begin{array}{l}8 \\
8\end{array}$ & $\begin{array}{l}40 \\
60\end{array}$ & $\begin{array}{l}\text { Waste paper recycling } \\
\text { Detergent industries }\end{array}$ & [111] \\
\hline Compost metagenome & E. coli & $\mathrm{CH} 1$ & 445 & 52 & 5.5 & 75 & $\begin{array}{l}\text { Biofuel production } \\
\text { Oligosaccharide synthesis }\end{array}$ & [108] \\
\hline Termite gut & E. coli & $\mathrm{CH} 1$ & 455 & 51 & 6.0 & 90 & Biofuel production & {$[105]$} \\
\hline Environmental DNA & E. coli & $\mathrm{CH} 1$ & 485 & 55 & 6.5 & 55 & $\begin{array}{l}\text { Ginsenoside conversion } \\
\text { Biofuel production }\end{array}$ & [49] \\
\hline Cow rumen metagenome & E. coli & $\mathrm{CH} 3$ & NR & NR & 5.0 & 50 & Biofuel production & {$[110]$} \\
\hline Cattle rumen metagenome & E. coli & $\mathrm{CH} 3$ & 779 & 85 & 5.0 & 38 & Biofuel production & {$[109]$} \\
\hline Bioreactor contents & E. coli & & 620 & 66 & 7.0 & 37 & Food Technology & {$[104]$} \\
\hline Mangrove metagenome & E. coli & $\mathrm{CH} 3$ & 663 & 71 & 7.0 & 40 & $\begin{array}{l}\text { Conversion of polydatin } \\
\text { to resveratrol }\end{array}$ & {$[251]$} \\
\hline Mangrove metagenome & E. coli & $\mathrm{CH} 3$ & 442 & 48 & 6.0 & 40 & Isoflavone hydrolysis & {$[140]$} \\
\hline
\end{tabular}

a.a= amino acids, $N R=$ not reported. 


\section{Cloning and Expression of Microbial $\beta$-Glucosidases}

Generally, cloning and expression of microbial genes encoding for industrially important enzymes aims to: i) produce enzymes in industrially compatible microorganisms such as Aspergillus, and Trichoderma species, if it is difficult to grow or handle the original microbes, ii) enhance enzyme production by expressing it in highly efficient host, using either multiple gene copies, and/or strong promoter, iii) produce enzymes in safe host if the origin of the genes is pathogenic or toxin producing microorganism, and iv) improve enzymes specificity, and stability by genetic engineering e.g., mutagenesis and direct evolution $[178,179]$. Number of $\beta$-glucosidase genes from bacteria, yeast and fungi have been cloned and expressed in E. coli and eukaryotic systems such as Saccharomyces cerevisiae, Pichia pastoris, and T. reesei [168,180,181,182]. Cloning and expression has started with either 1) genomic DNA digestion, construction of genomic DNA library and then functional screening for $\beta$-glucosidase [183], or 2) construction of cDNA library and function-based screening of $\beta$-glucosidase $[169,184]$.

Filamentous fungi are known to synthesize and secrete high amounts of extracellular enzymes including $\beta$-glucosidase and reports on cloning and expression of $\beta$-glucosidase genes from filamentous fungi are quiet less [185]. In addition, fungal genes complexity e.g., presence of introns, and post-transcriptional and post-translational modifications such as glycosylation, acts as hurdle for cloning of these genes [186]. Nevertheless, $\beta$-glucosidase genes from T. emersonii, P. brasilianum and T. aurantiacus have been cloned and expressed in filamentous fungi such as T. reesei [187], A. oryzae [183], and P. pastoris [43], respectively. $P$. pastoris is known to secrete high amount of extracellular protein therefore it is preferred for heterologous expression of recombinant proteins $[188,189]$. Similarly, $T$. reesei produces cellulase component (endoglucanase and exoglucanase) in high amount with excellent properties such as thermostability and catalytic efficiency but it lacks sufficient amount of $\beta$-glucosidase. Researchers therefore have heterologously expressed exogenous $\beta$-glucosidase genes in $T$. reesei under strong promoter in order to enhance its $\beta$-glucosidase activity so that cellulose hydrolysis rate was improved considerably [112,187,190,191,192]. Majority of recombinant fungal $\beta$-glucosidase belong to glycoside hydrolase family 3 . Fungal species are usually transformed with linearized plasmid which integrates to the chromosomal DNA enhancing its stability and expression efficiency $[187,193]$.

Bacterial $\beta$-glucosidase genes has been cloned from number of species and expressed in E. coli because of its high growth rate, easy handling, genetic simplicity, easy transformation and plasmid uptake, and it can grow to high cell density 200 g/1 [194,195]. However, expression in $E$. coli has several drawbacks such as formation of inclusion bodies, low secretion efficiency, absence of splicing machinery and inability to perform post-translational modifications such as glycosylation explaining why it is not successfully being used for expression of fungal $\beta$-glucosidase enzymes [196,197,198]. E.coli is usually transformed with self-replicated plasmid that does not integrate to chromosomal DNA and keeps on replicating itself independently from cell divisions [199,200].

Finally, metagenome represents an excellent cultureindependent approach for obtaining industrially important enzymes from unculturable microorganisms. A number of $\beta$-glucosidase has been cloned, expressed and characterized from different environment such as cattle rumen, marine, compost, soil metagenomes etc. In this approach, environmental DNA is first extracted and digested. The digested DNA is ligated into a particular vector for construction of metagenomic library which is then screened using function-based approach. List of recombinant $\beta$-glucosidase from fungi, bacteria and metagenome sources are shown in Table 2.

\section{Applications of $\beta$-Glucosidase}

$\beta$-Glucosidases are a group of enzymes that catalyze the hydrolysis of nonreducing $\beta$-glucosyl terminal from wide variety of aryl, alkyl glycosides, disaccharides and cellooligosaccharides. It is a component of cellulase system involving in hydrolysis of cellobiose and short oligosaccharides to glucose thus eliminating the inhibitory effect of cellobiose on both endo- and exo-glucanases [201]. In addition to hydrolytic activity, $\beta$-glucosidase, under certain circumstances, have synthetic activity via transglycosylation [24,202]. As a result, $\beta$-glucosidase has wide range of potential biotechnological applications, some of which are based on the hydrolytic activity and some are based on the synthetic activity.

\subsection{Applications Based on Hydrolytic Activity}

Biofuel production from lignocellulose material is very attractive area for research in the current era especially with arising of energy crisis, depletion of fossil fuel, energy high prices and global warming. $\beta$-Glucosidase is utilized in hydrolysis of cellobiose and short oligosaccharides to glucose during cellulose hydrolysis so that glucose can be fermented to ethanol or other biofuel $[13,28,128]$. Currently, $T$. reesei is the major source of the commercial cellulase enzyme used for cellulose hydrolysis and biofuel production but it lacks sufficient $\beta$-glucosidase activity therefore supplementation of exogenous $\beta$-glucosidase from other filamentous fungi such as Aspergillus niger is mandatory [28].

$\beta$-Glucosidase is also utilized in aroma enhancement of wine and fruit juices by liberation of aromatic compounds from their glycosidic precursors present in fruit juices, musts and wines [203]. Since endogenous plant $\beta$-glucosidase is less efficient, supplementation with exogenous enzyme from microbial sources enhances the release of aromatic compounds from their glyosidic precursors [204,205].

$\beta$-Glucosidase is also used in the hydrolysis of phenolic compounds such as flavonoids and isoflavone glycosides present in soybean, fruit, vegetables, and other plant-derived foods [156,206,207,208]. These compounds are known to have phytoestrogenic [209], antioxidant [210,211], anticancer [212], and antinflammatory activities [213]. However, they are present mainly in glycosides 
form thus decreasing their absorption from small intestine. As a result, hydrolysis of these phenolic glycosides by $\beta$-glucosidases increases their absorption and bioavailability [211,214]. These compounds may include diadzin, genistin, sesaminol glycosides, hesperidin, neohesperidin, naringin, narirutin, rutin, neoponcirin [61,211,215].

$\beta$-Glucosidases can also be used for debittering of fruit juices by hydrolysis of bitter compounds such naringenin and oleuropein [215,216]. Another important application of $\beta$-glucosidase is cassava detoxification, a carbohydrate rich food and a staple food in tropical countries. Because cassava is known to contain toxic cyanogenic glycosides such as linamarin and lotaustralin, prolonged consumption of cassava has been associated with CNS syndrome "Konzo" [217,218]. These cyanogenic glycosides can be eliminated with addition of exogenous linamarase and $\beta$-glucosidase from microbial sources during cassava processing [22,29,219]. Finally, $\beta$-glucosidase, along with other cellulases and hemicellulases enzymes, is utilized in waste paper recycling and ink removal for production of biofuel [1,30,220,221].

\subsection{Applications Based on Synthetic Activity}

The synthetic activity of $\beta$-glucosidase can be invested for synthesis of different alkyl- and aryl- $\beta$-glycosides and oligosaccharides. Alkyl- and aryl- $\beta$-glycosides have a wide range of applications in pharmaceutical and medical sciences increasing the demand of these valuable compounds. The enzymatic methods for synthesis of such compounds provides the advantage of high regio- and stereo-selectivity and utilization of mild conditions over the chemical methods which are nonspecific and require very harsh conditions [143]. Alkyl polyglucosides (APGs) are used for improving lubricating properties of water since they are biodegradable and have surface activity. Alkyl- and aryl- $\beta$-glycosides are nonionic surfactants with chemical stability, safety, biogradability, and antimicrobial activity. They can be used in detergent, pharmaceutical, food, and cosmetics industries [222]. On the other hand, synthetic oligosaccharides have broad spectrum of applications particularly in biomedical science as therapeutic agents; growth promoting agents for probiotics bacteria, vaccines, and diagnostic tools [35]. Synthetic Galacto-oligosaccharides (GOS) produced from lactose have a fiber properties relieving the symptoms of constipation in adult populations and modulate bowel function and stool characters [223,224]. GOS supplemented in infant formula stimulate intestinal Bifidobacteria and Lactobacilli to the same extent the breast-fed infants could have [202,225,226].

\section{Conclusion}

$\beta$-Glucosidases are heterogeneous group of enzymes found in bacteria, fungi, plants and animals. $\beta$ Glucosidases are diverse enzymes exhibiting different $\mathrm{pH}$ and temperature optima, substrate specificity, localization, and biological roles. $\beta$-Glucosidases have broad spectrum of potential biotechnological applications particularly in biofuel production, isoflavone hydrolysis, bitterness removal, cassava detoxification, etc. These potential applications call for effective large scale production of this valuable enzyme from fungal, bacterial and metagenome sources. Moreover, application of $\beta$-glucosidases in biofuel production require a novel enzymes exhibiting high thermostability, acidophilicity, and glucose tolerance therefore the search for enzymes exhibiting high tolerance to temperature, acidic $\mathrm{pH}$ and high glucose concentration is highly encouraged. Synthetic activity of $\beta$-glucosidases through transglycosylation is another attractive activity in biomedical and pharmaceutical applications and the search for new $\beta$-glucosidases with an excellent transglycosylation activity is another avenue of research in this field.

\section{References}

[1] Ahmed A, Nasim Fu-H, Batool K, Bibi A. Microbial $\beta$-Glucosidase: Sources, Production and Applications. Journal of Applied \& Environmental Microbiology 2017, 5(1): 31-46.

[2] Acharya S, Chaudhary A. Bioprospecting thermophiles for cellulase production: a review. Brazilian Journal of Microbiology 2012, 43(3): 844-856.

[3] Anwar Z, Gulfraz M, Irshad M. Agro-industrial lignocellulosic biomass a key to unlock the future bio-energy: A brief review. Journal of Radiation Research and Applied Sciences 2014, 7(2): 163-173.

[4] Persson I, Tjerneld F, Hahn-Hägerdal B. Fungal cellulolytic enzyme production: A review. Process Biochemistry 1991, 26(2): 65-74.

[5] Pérez J, Munoz-Dorado J, de la Rubia T, Martinez J. Biodegradation and biological treatments of cellulose, hemicellulose and lignin: an overview. International Microbiology 2002, 5(2): 53-63.

[6] Horn SJ, Vaaje-Kolstad G, Westereng B, Eijsink VGH. Novel enzymes for the degradation of cellulose. Biotechnology for Biofuels 2012, 5: 45-45.

[7] Béguin P, Aubert J-P. The biological degradation of cellulose. FEMS Microbiology Reviews 1994, 13(1): 25-58.

[8] Mussatto SI, Teixeira J. Lignocellulose as raw material in fermentation processes. Current Research, Technology and Education Topics in Applied Microbiology and Microbial Biotechnology (Méndez-Vilas, A, Ed) 2010, 2: 897-907.

[9] Cragg SM, Beckham GT, Bruce NC, Bugg TD, Distel DL, Dupree P, Etxabe AG, Goodell BS, Jellison J, McGeehan JE. Lignocellulose degradation mechanisms across the Tree of Life. Current Opinion in Chemical Biology 2015, 29: 108-119.

[10] Tiwari P, Misra B, Sangwan NS. $\beta$-Glucosidases from the fungus Trichoderma: an efficient cellulase machinery in biotechnological applications. BioMed Research International 2013, 2013.

[11] Singh G, Verma A, Kumar V. Catalytic properties, functional attributes and industrial applications of $\beta$-glucosidases. 3 Biotech 2016, 6(1): 1-14.

[12] Bhatia Y, Mishra S, Bisaria V. Microbial $\beta$-glucosidases: cloning, properties, and applications. Critical Reviews in Biotechnology 2002, 22(4): 375-407.

[13] Teugjas H, Väljamäe P. Selecting $\beta$-glucosidases to support cellulases in cellulose saccharification. Biotechnology for Biofuels 2013, 6(1): 1.

[14] Cantarel BL, Coutinho PM, Rancurel C, Bernard T, Lombard V, Henrissat B. The Carbohydrate-Active EnZymes database (CAZy) an expert resource for Glycogenomics. Nucleic Acids Research 2009, 37(Database issue): D233-D238.

[15] Cairns JRK, Esen A. $\beta$-Glucosidases. Cellular and Molecular Life Sciences 2010, 67(20): 3389-3405.

[16] Singh G, Verma AK, Kumar V. Catalytic properties, functional attributes and industrial applications of $\beta$-glucosidases. 3 Biotech 2016, 6(1): 3.

[17] Lieberman RL, Wustman BA, Huertas P, Powe AC, Pine CW Khanna R, Schlossmacher MG, Ringe D, Petsko GA. Structure of acid [beta]-glucosidase with pharmacological chaperone provides insight into Gaucher disease. Nature Chemical Biology 2007, 3(2): 101-107. 
[18] Wang Y, Chantreau M, Sibout R, Hawkins S. Plant cell wall lignification and monolignol metabolism. Frontiers in Plant Science 2013, 4: 220.

[19] Stanley D, Rejzek M, Naested H, Smedley M, Otero S, Fahy B, Thorpe F, Nash RJ, Harwood W, Svensson B et al. The Role of $\alpha$-Glucosidase in Germinating Barley Grains. Plant Physiology 2011, 155(2): 932-943.

[20] Dietz KJ, Sauter A, Wichert K, Messdaghi D, Hartung W. Extracellular $\beta$-glucosidase activity in barley involved in the hydrolysis of ABA glucose conjugate in leaves. Journal of Experimental Botany 2000, 51(346): 937-944.

[21] Barleben L, Panjikar S, Ruppert M, Koepke J, Stöckigt J. Molecular Architecture of Strictosidine Glucosidase: The Gateway to the Biosynthesis of the Monoterpenoid Indole Alkaloid Family. The Plant Cell 2007, 19(9): 2886-2897.

[22] Eksittikul T, Chulavatnatol M. Characterization of cyanogenic $\beta$-glucosidase (linamarase) from cassava (Manihot esculenta Crantz). Archives of Biochemistry and Biophysics 1988, 266(1): 263-269.

[23] Balmer D, Flors V, Glauser G, Mauch-Mani B. Metabolomics of cereals under biotic stress: current knowledge and techniques. Frontiers in Plant Science 2013, 4: 82.

[24] Singhania RR, Patel AK, Sukumaran RK, Larroche C, Pandey A. Role and significance of beta-glucosidases in the hydrolysis of cellulose for bioethanol production. Bioresource Technology 2013, 127: 500-507.

[25] Fowler T, Brown RD. The bgI1 gene encoding extracellular $\beta$-glucosidase from Trichoderma reesei is required for rapid induction of the cellulase complex. Molecular Microbiology 1992, 6(21): 3225-3235.

[26] Faure D. The family-3 glycoside hydrolases: from housekeeping functions to host-microbe interactions. Applied and Environmental Microbiology 2002, 68(4): 1485-1490.

[27] Michlmayr H, Kneifel W. $\beta$-Glucosidase activities of lactic acid bacteria: mechanisms, impact on fermented food and human health. FEMS Microbiology Letters 2014, 352(1): 1-10.

[28] Sørensen A, Lübeck M, Lübeck PS, Ahring BK. Fungal betaglucosidases: a bottleneck in industrial use of lignocellulosic materials. Biomolecules 2013, 3(3): 612-631.

[29] Gueguen Y, Chemardin P, Labrot P, Arnaud A, Galzy P. Purification and characterization of an intracellular $\beta$-glucosidase from a new strain of Leuconostoc mesenteroides isolated from cassava. Journal of Applied Microbiology 1997, 82(4): 469-476.

[30] Elliston A, Collins SR, Faulds CB, Roberts IN, Waldron KW. Biorefining of waste paper biomass: Increasing the concentration of glucose by optimising enzymatic hydrolysis. Applied Biochemistry and Biotechnology 2014, 172(7): 3621-3634.

[31] Bohlin C, Praestgaard E, Baumann MJ, Borch K, Praestgaard J, Monrad RN, Westh P. A comparative study of hydrolysis and transglycosylation activities of fungal $\beta$-glucosidases. Applied Microbiology and Biotechnology 2013, 97(1): 159-169.

[32] Driguez P-A, Potier P, Trouilleux P. Synthetic oligosaccharides as active pharmaceutical ingredients: Lessons learned from the full synthesis of one heparin derivative on a large scale. Natural Product Reports 2014, 31(8): 980-989.

[33] Pozsgay V. Recent developments in synthetic oligosaccharidebased bacterial vaccines. Current Topics in Medicinal Chemistry 2008, 8(2): 126-140.

[34] Boltje TJ, Buskas T, Boons G-J. Opportunities and challenges in synthetic oligosaccharide and glycoconjugate research. Nature Chemistry 2009, 1(8): 611-622.

[35] Seeberger PH, Werz DB. Synthesis and medical applications of oligosaccharides. Nature 2007, 446(7139): 1046-1051.

[36] Veena V, Poornima P, Parvatham R, Kalaiselvi K. Isolation and characterization of $\beta$-glucosidase producing bacteria from different sources. African Journal of Biotechnology 2011, 10(66): 14891-14906.

[37] Daenen L, Saison D, Sterckx F, Delvaux F, Verachtert H, Derdelinckx G. Screening and evaluation of the glucoside hydrolase activity in Saccharomyces and Brettanomyces brewing yeasts. Journal of Applied Microbiology 2008, 104(2): 478-488.

[38] Daoust RA, Litsky W. Pfizer selective enterococcus agar overlay method for the enumeration of fecal streptococci by membrane filtration. Applied Microbiology 1975, 29(5): 584-589.

[39] James AL, Yeoman P. Detection of specific bacterial enzymes by high contrast metal chelate formation Part I. 8-Hydroxyquinoline-
$\beta$-D-glucoside, an alternative to aesculin in the differentation of members of the familiy Enterobacteriaceae. Zentralblatt für Bakteriologie, Mikrobiologie und Hygiene Series A 1987, 267(2): 188-193.

[40] James A, Perry J, Ford M, Armstrong L, Gould F. Note: Cyclohexenoesculetin- $\beta$-D-glucoside: a new substrate for the detection of bacterial $\beta$-D-glucosidase. Journal of Applied Microbiology 1997, 82(4): 532-536.

[41] Mendes Ferreira A, Climaco M, Mendes Faia A. The role of non-Saccharomyces species in releasing glycosidic bound fraction of grape aroma components-a preliminary study. Journal of Applied Microbiology 2001, 91(1): 67-71.

[42] Gao Z, Van Hop D, Yen LTH, Ando K, Hiyamuta S, Kondo R. The production of $\beta$-glucosidases by Fusarium proliferatum NBRC109045 isolated from Vietnamese forest. AMB Express 2012, 2: 49-49.

[43] Hong J, Tamaki H, Kumagai H. Cloning and functional expression of thermostable $\beta$-glucosidase gene from Thermoascus aurantiacus. Applied Microbiology and Biotechnology 2007, 73(6): 1331-1339.

[44] Du J, Cui C-H, Park SC, Kim J-K, Yu H-S, Jin F-X, Sun C, Kim $\mathrm{S}-\mathrm{C}, \quad \mathrm{Im} \mathrm{W}-\mathrm{T}$. Identification and Characterization of a Ginsenoside-Transforming $\beta$-Glucosidase from Pseudonocardia sp. Gsoil 1536 and Its Application for Enhanced Production of Minor Ginsenoside Rg 2 (S). PloS one 2014, 9(6): e96914.

[45] Uchiyama T, Yaoi K, Miyazaki K. Glucose-tolerant $\beta$-glucosidase retrieved from a Kusaya gravy metagenome. Frontiers in Microbiology 2015, 6: 548.

[46] Perry J, Morris K, James A, Oliver M, Gould FK. Evaluation of novel chromogenic substrates for the detection of bacterial $\beta$-glucosidase. Journal of Applied Microbiology 2007, 102(2): 410-415.

[47] Liu W, Hong J, Bevan DR, Zhang YHP. Fast identification of thermostable beta-glucosidase mutants on cellobiose by a novel combinatorial selection/screening approach. Biotechnology and Bioengineering 2009, 103(6): 1087-1094.

[48] Acker MG, Auld DS. Considerations for the design and reporting of enzyme assays in high-throughput screening applications. Perspectives in Science 2014, 1(1-6): 56-73.

[49] Kim S-J, Lee C-M, Kim M-Y, Yeo Y-S, Yoon S-H, Kang H-C, Koo B-S. Screening and characterization of an enzyme with betaglucosidase activity from environmental DNA. Journal of Microbiology and Biotechnology 2007, 17(6): 905-912.

[50] Li Y-Y, Jiang C-J, Wan X-C, Zhang Z-Z, Li D-X. Purification and partial characterization of $\beta$-glucosidase from fresh leaves of tea plants (Camellia sinensis (L.) O. Kuntze). Acta Biochimica et Biophysica Sinica 2005, 37(6): 363-370.

[51] Olajuyigbe FM, Nlekerem CM, Ogunyewo OA. Production and Characterization of Highly Thermostable $\beta$-Glucosidase during the Biodegradation of Methyl Cellulose by Fusarium oxysporum. Biochemistry Research International 2016, 2016.

[52] Rajoka MI, Akhtar MW, Hanif A, Khalid A. Production and characterization of a highly active cellobiase from Aspergillus niger grown in solid state fermentation. World Journal of Microbiology and Biotechnology 2006, 22(9): 991-998.

[53] Cao L-c, Wang Z-j, Ren G-h, Kong W, Li L, Xie W, Liu Y-h. Engineering a novel glucose-tolerant $\beta$-glucosidase as supplementation to enhance the hydrolysis of sugarcane bagasse at high glucose concentration. Biotechnology for Biofuels 2015, 8: 202.

[54] Goswami S, Gupta N, Datta S. Using the $\beta$-glucosidase catalyzed reaction product glucose to improve the ionic liquid tolerance of $\beta$-glucosidases. Biotechnology for Biofuels 2016, 9(1): 1.

[55] Bai H, Wang H, Sun J, Irfan M, Han M, Huang Y, Han X, Yang Q Production, purification and characterization of novel beta glucosidase from newly isolated Penicillium simplicissimum H-11 in submerged fermentation. EXCLI Journal 2013, 12: 528.

[56] Cao P, Wang L, Wang Y, Zhou N, Chen Y. Alkali-tolerant $\beta$ glucosidase produced by newly isolated Aspergillus fumigatus WL002 from rotten wood. International Biodeterioration \& Biodegradation 2015, 105: 276-282.

[57] Setlow B, Cabrera-Martinez RM, Setlow P. Mechanism of the hydrolysis of 4-methylumbelliferyl- $\beta$-d-glucoside by germinating and outgrowing spores of Bacillus species. Journal of Applied Microbiology 2004, 96(6): 1245-1255.

[58] YANG L, AKAO T, KOBASHI K, HATTORI M. Purification and Characterization of a Novel Sennoside-Hydrolyzing. BETA.- 
Glucosidase from Bifidobacterium Sp. Strain SEN, a Human Intestinal Anaerobe. Biological and Pharmaceutical Bulletin 1996, 19(5): 705-709.

[59] Yang S, Jiang Z, Yan Q, Zhu H. Characterization of a thermostable extracellular $\beta$-glucosidase with activities of exoglucanase and transglycosylation from Paecilomyces thermophila. Journal of Agricultural and Food Chemistry 2007, 56(2): 602-608.

[60] Yeom S-J, Kim B-N, Kim Y-S, Oh D-K. Hydrolysis of isoflavone glycosides by a thermostable $\beta$-glucosidase from Pyrococcus furiosus. Journal of Agricultural and Food Chemistry 2012, 60(6): 1535-1541.

[61] Yang S, Wang L, Yan Q, Jiang Z, Li L. Hydrolysis of soybean isoflavone glycosides by a thermostable $\beta$-glucosidase from Paecilomycesthermophila. Food Chemistry 2009, 115(4): 1247-1252.

[62] Kimura I, Yoshioka N, Tajima S. Purification and characterization of a $\beta$-glucosidase with $\beta$-xylosidase activity from aspergillus sojae. Journal of Bioscience and Bioengineering 1999, 87(4): 538-541.

[63] Kengen SW, Luesink EJ, STAMS AJ, ZEHNDER AJ. Purification and characterization of an extremely thermostable $\beta$-glucosidase from the hyperthermophilic archaeon Pyrococcus furiosus. European Journal of Biochemistry 1993, 213(1): 305-312.

[64] Wierzbicka-Woś A, Bartasun P, Cieśliński H, Kur J. Cloning and characterization of a novel cold-active glycoside hydrolase family 1 enzyme with $\beta$-glucosidase, $\beta$-fucosidase and $\beta$-galactosidase activities. BMC Biotechnology 2013, 13(1): 1.

[65] Karnchanatat A, Petsom A, Sangvanich P, Piaphukiew J, Whalley AJ, Reynolds CD, Sihanonth P. Purification and biochemical characterization of an extracellular $\beta$-glucosidase from the wooddecaying fungus Daldinia eschscholzii (Ehrenb.: Fr.) Rehm. FEMS Microbiology Letters 2007, 270(1): 162-170.

[66] Yoon J-J, Kim K-Y, Cha C-J. Purification and characterization of thermostable $\beta$-glucosidase from the brown-rot basidiomycete Fomitopsis palustris grown on microcrystalline cellulose. The Journal of Microbiology 2008, 46(1): 51-55.

[67] Yang L, Ning ZS, Shi CZ, Chang ZY, Huan LY. Purification and characterization of an isoflavone-conjugates-hydrolyzing $\beta$ glucosidase from endophytic bacterium. Journal of Agricultural and Food Chemistry 2004, 52(7): 1940-1944.

[68] Zhang Y, Yuan L, Chen Z, Fu L, Lu J, Meng Q, He H, Yu X, Lin F, Teng L. Purification and characterization of beta-glucosidase from a newly isolated strain Tolypocladium cylindrosporum Syzx4. Chemical Research in Chinese Universities 2009, 27. 557-561.

[69] Gong G, Zheng Z, Liu H, Wang L, Diao J, Wang P, Zhao G Purification and characterization of a $\beta$-glucosidase from Aspergillus niger and its application in the hydrolysis of geniposide to genipin. Journal of Microbiology and Biotechnology 2014, 24(6): 788-794.

[70] Lin J, Pillay B, Singh S. Purification and biochemical characteristics of $\beta$-D-glucosidase from a thermophilic fungus, Thermomyces lanuginosus-SSBP. Biotechnology and Applied Biochemistry 1999, 30(1): 81-87.

[71] Bhat KM, Gaikwad JS, Maheshwari R. Purification and characterization of an extracellular $\beta$-glucosidase from the thermophilic fungus Sporotrichum thermophile and its influence on cellulase activity. Microbiology 1993, 139(11): 2825-2832.

[72] Liu D, Zhang R, Yang X, Zhang Z, Song S, Miao Y, Shen Q. Characterization of a thermostable $\beta$-glucosidase from Aspergillus fumigatus Z5, and its functional expression in Pichia pastoris X33. Microbial Cell Factories 2012, 11(1): 1.

[73] Narasimha G, Sridevi A, Ramanjaneyulu G, Rajasekhar Reddy B. Purification and Characterization of $\beta$-Glucosidase from Aspergillus niger. International Journal of Food Properties 2016, 19(3): 652-661.

[74] Park A-R, Hong JH, Kim J-J, Yoon J-J. Biochemical characterization of an extracellular $\beta$-glucosidase from the fungus, Penicillium italicum, isolated from rotten citrus peel. Mycobiology 2012, 40(3): 173-180.

[75] Venturi LL, de Lourdes Polizeli M, Terenzi HF, dos Prazeres Melo Furriel R, Jorge JA. Extracellular $\beta$-D-glucosidase from Chaetomium thermophilum var. coprophilum: production, purification and some biochemical properties. Journal of Basic Microbiology 2002, 42(1): 55.
[76] De Palma-Fernandez E, Gomes E, Da Silva R. Purification and characterization of two $\beta$-glucosidases from the thermophilic fungusThermoascus aurantiacus. Folia Microbiologica 2002, 47(6): 685-690.

[77] Ranjitha P, Karthy E, Mohankumar A. Purification and Characterization of the Lipase from Marine Vibrio fischeri. International Journal of Biology 2009, 1(2): 48.

[78] Zaidi KU, Ali AS, Ali SA. Purification and characterization of melanogenic enzyme tyrosinase from button mushroom. Enzyme Research 2014, 2014.

[79] Karnaouri A, Topakas E, Paschos T, Taouki I, Christakopoulos P. Cloning, expression and characterization of an ethanol tolerant GH3 $\beta$-glucosidase from Myceliophthora thermophila. PeerJ 2013 1: e46.

[80] Saha BC, Freer SN, Bothast RJ. Production, purification, and properties of a thermostable $\beta$-glucosidase from a color variant strain of Aureobasidium pullulans. Applied and Environmental Microbiology 1994, 60(10): 3774-3780.

[81] Hernández-Guzmán A, Flores-Martínez A, Ponce-Noyola P, Villagómez-Castro JC. Purification and characterization of an extracellular $\beta$-glucosidase from Sporothrix schenckii. FEBS Open Bio 2016, 6(11): 1067-1077.

[82] Bornhorst JA, Falke JJ. Purification of proteins using polyhistidine affinity tags. Methods in Enzymology 2000, 326: 245-254.

[83] Chan CS, Sin LL, Chan K-G, Shamsir MS, Manan FA, Sani RK, Goh KM: Characterization of a glucose-tolerant $\beta$-glucosidase from Anoxybacillus sp. DT3-1. Biotechnology for Biofuels 2016, 9(1): 174

[84] Crespim E, Zanphorlin LM, de Souza FH, Diogo JA, Gazolla AC, Machado CB, Figueiredo F, Sousa AS, Nóbrega F, Pellizari VH: A novel cold-adapted and glucose-tolerant GH1 $\beta$-glucosidase from Exiguobacterium antarcticum B7. International Journal of Biological Macromolecules 2016, 82: 375-380.

[85] Pei J, Pang Q, Zhao L, Fan S, Shi H. Thermoanaerobacterium thermosaccharolyticum $\beta$-glucosidase: a glucose-tolerant enzyme with high specific activity for cellobiose. Biotechnology for Biofuels 2012, 5(1): 1.

[86] Kalyani D, Lee K-M, Tiwari MK, Ramachandran P, Kim H, Kim $\mathrm{I}-\mathrm{W}$, Jeya M, Lee J-K. Characterization of a recombinant aryl $\beta$ glucosidase from Neosartorya fischeri NRRL181. Applied Microbiology and Biotechnology 2012, 94(2): 413-423.

[87] Shipkowski S, Brenchley JE. Characterization of an unusual cold-active $\beta$-glucosidase belonging to family 3 of the glycoside hydrolases from the psychrophilic isolate Paenibacillus sp. strain C7. Applied and Environmental Microbiology 2005, 71(8): 4225-4232.

[88] Harper S, Speicher DW. Purification of proteins fused to glutathione S-tranferase. Methods in Molecular Biology (Clifton, NJ) 2011, 681: 259-280.

[89] Chen S, Hong Y, Shao Z, Liu Z. A cold-active $\beta$-glucosidase (Bgl1C) from a sea bacteria Exiguobacterium oxidotolerans A011. World Journal of Microbiology and Biotechnology 2010, 26(8): 1427-1435.

[90] Laderman KA, Davis BR, Krutzsch HC, Lewis MS, Griko Y, Privalov PL, Anfinsen CB. The purification and characterization of an extremely thermostable alpha-amylase from the hyperthermophilic archaebacterium Pyrococcus furiosus. Journal of Biological Chemistry 1993, 268(32): 24394-24401.

[91] Xian L, Wang F, Luo X, Feng Y-L, Feng J-X. Purification and Characterization of a Highly Efficient Calcium-Independent $\alpha$-Amylase from Talaromyces pinophilus $1-95$. PloS one 2015, 10(3): e0121531.

[92] Kamaruddin S, Bakar FDA, Illias R, Said M, Hassan O, Murad AMA. Overexpression, purification and characterization of Aspergillus niger Beta-Glucosidase in Pichia pastoris. Malaysian Applied Biology 2015, 44(1): 7-11

[93] Daroit DJ, Simonetti A, Hertz PF, Brandelli A. Purification and characterization of an extracellular beta-glucosidase from Monascus purpureus. Journal of Microbiology and Biotechnology 2008, 18(5): 933-941.

[94] Elíades LA, Rojas NL, Cabello MN, Voget CE, Saparrat MCN. $\alpha$-L-Rhamnosidase and $\beta$-D-glucosidase activities in fungal strains isolated from alkaline soils and their potential in naringin hydrolysis. Journal of Basic Microbiology 2011, 51(6): 659-665.

[95] Ravindran C, Varatharajan GR, Karthikeyan A. Role of alkalinetolerant fungal cellulases in release of total antioxidants from 
agro-wastes under solid state fermentation. 2011, BioResource. 6, 3142-3154.

[96] An D-S, Cui C-H, Lee H-G, Wang L, Kim SC, Lee S-T, Jin F, Yu $\mathrm{H}$, Chin $\mathrm{Y}-\mathrm{W}$, Lee H-K. Identification and characterization of a novel Terrabacter ginsenosidimutans sp. nov. $\beta$-glucosidase that transforms ginsenoside Rb1 into the rare gypenosides XVII and LXXV. Applied and Environmental Microbiology 2010, 76(17): 5827-5836.

[97] Zahoor S, Javed MM, Aftab MN, Ikram-ul-Haq. Cloning and expression of $\beta$-glucosidase gene from Bacillus licheniformis into E. coli BL 21 (DE3). Biologia 2011, 66(2): 213-220.

[98] Hong M-R, Kim Y-S, Park C-S, Lee J-K, Kim Y-S, Oh D-K. Characterization of a recombinant $\beta$-glucosidase from the thermophilic bacterium Caldicellulosiruptor saccharolyticus. Journal of Bioscience and Bioengineering 2009, 108(1): 36-40.

[99] Naz S, Ikram N, Rajoka M, Sadaf S, Akhtar M. Enhanced production and characterization of a $\beta$-glucosidase from Bacillus halodurans expressed in Escherichia coli. Biochemistry (Moscow) 2010, 75(4): 513-518.

[100] Ma Z, Lan X, Tang J, Xu J, Xie J, Chen Q, Pan G, Li C, Zhou Z. Molecular cloning and characterization of a novel alkaline $\beta$-glucosidase encoding gene (kpYBDN) from Klebsiella pneumoniae, performing a conserved function in bacteria. International Journal of Agriculture and Biology 2015, 17(5): 983-989.

[101] Saha BC, Bothast RJ: Production, purification, and characterization of a highly glucose-tolerant novel betaglucosidase from Candida peltata. Applied and Environmental Microbiology 1996, 62(9): 3165-3170.

[102] Rajoka M, Khan S, Latif F, Shahid R: Influence of carbon and nitrogen sources and temperature on hyperproduction of a thermotolerant $\beta$-glucosidase from synthetic medium by Kluyveromyces marxianus. Applied Biochemistry and Biotechnology 2004, 117(2): 75-92.

[103] Iembo T, Da Silva R, Pagnocca F, Gomes E. Production, Characterization, and Properties of $\beta$-Glucosidase and $\beta$-Xylosidase from a Strain of Aureobasidium sp. Applied Biochemistry and Microbiology 2002, 38(6): 549-552.

[104] Jiang C, Hao Z-Y, Jin K, Li S-X, Che Z-Q, Ma G-F, Wu B. Identification of a metagenome-derived $\beta$-glucosidase from bioreactor contents. Journal of Molecular Catalysis B: Enzymatic 2010, 63(1): 11-16.

[105] Wang Q, Qian C, Zhang X-Z, Liu N, Yan X, Zhou Z. Characterization of a novel thermostable $\beta$-glucosidase from a metagenomic library of termite gut. Enzyme and Microbial Technology 2012, 51(6): 319-324.

[106] Lu J, Du L, Wei Y, Hu Y, Huang R. Expression and characterization of a novel highly glucose-tolerant $\beta$-glucosidase from a soil metagenome. Acta Biochimica et Biophysica Sinica 2013, 45, 664-73.

[107] Schröder C, Elleuche S, Blank S, Antranikian G. Characterization of a heat-active archaeal $\beta$-glucosidase from a hydrothermal spring metagenome. Enzyme and Microbial Technology 2014, 57: 48-54.

[108] Uchiyama T, Miyazaki K, Yaoi K. Characterization of a novel $\beta$ glucosidase from a compost microbial metagenome with strong transglycosylation activity. Journal of Biological Chemistry 2013, 288(25): 18325-18334.

[109] Li Y, Liu N, Yang H, Zhao F, Yu Y, Tian Y, Lu X. Cloning and characterization of a new $\beta$-Glucosidase from a metagenomic library of Rumen of cattle feeding with Miscanthus sinensis. BMC Biotechnology 2014, 14(1): 1.

[110] Del Pozo MV, Fernández-Arrojo L, Gil-Martínez J, Montesinos A, Chernikova TN, Nechitaylo TY, Waliszek A, Tortajada M, Rojas A, Huws SA. Microbial $\beta$-glucosidases from cow rumen metagenome enhance the saccharification of lignocellulose in combination with commercial cellulase cocktail. Biotechnology for Biofuels 2012, 5(1): 1.

[111] Biver S, Stroobants A, Portetelle D, Vandenbol M. Two promising alkaline $\beta$-glucosidases isolated by functional metagenomics from agricultural soil, including one showing high tolerance towards harsh detergents, oxidants and glucose. Journal of Industrial Microbiology \& Biotechnology 2014, 41(3): 479-488.

[112] Jiang C, Li S-X, Luo F-F, Jin K, Wang Q, Hao Z-Y, Wu L-L, Zhao G-C, Ma G-F, Shen P-H. Biochemical characterization of two novel $\beta$-glucosidase genes by metagenome expression cloning. Bioresource Technology 2011, 102(3): 3272-3278.
[113] Mallerman J, Papinutti L, Levin L. Characterization of $\beta$ Glucosidase Produced by the White Rot Fungus Flammulina velutipes. Journal of Microbiology and Biotechnology 2015, 25(1) 57-65.

[114] Dhake A, Patil M. Production of ß-Glucosidase by Penicillium purpurogenum. Brazilian Journal of Microbiology 2005, 36(2): $170-176$.

[115] Magalhaes P, Ferraz A, Milagres A. Enzymatic properties of two $\beta$-glucosidases from Ceriporiopsis subvermispora produced in biopulping conditions. Journal of Applied Microbiology 2006, 101(2): 480-486.

[116] Hassan N, Nguyen T-H, Intanon M, Kori LD, Patel BK, Haltrich D, Divne C, Tan TC: Biochemical and structural characterization of a thermostable $\beta$-glucosidase from Halothermothrix orenii for galacto-oligosaccharide synthesis. Applied Microbiology and Biotechnology 2015, 99(4): 1731-1744.

[117] Chang KH, Jo MN, Kim K-T, Paik H-D. Purification and characterization of a ginsenoside Rb1-hydrolyzing $\beta$-glucosidase from Aspergillus niger KCCM 11239. International Journal of Molecular Sciences 2012, 13(9): 12140-12152.

[118] Chen H-L, Chen Y-C, Lu M-YJ, Chang J-J, Wang H-TC, Ke H-M, Wang T-Y, Ruan S-K, Wang T-Y, Hung K-Y. A highly efficient $\beta$-glucosidase from the buffalo rumen fungus Neocallimastix patriciarum W5. Biotechnology for Biofuels 2012, 5(1): 1.

[119] Jeya M, Joo A-R, Lee K-M, Tiwari MK, Lee K-M, Kim S-H, Lee $\mathrm{J}-\mathrm{K}$. Characterization of $\beta$-glucosidase from a strain of Penicillium purpurogenum KJS506. Applied Microbiology and Biotechnology 2010, 86(5): 1473-1484.

[120] Cristóbal HA, Schmidt A, Kothe E, Breccia J, Abate CM. Characterization of inducible cold-active $\beta$-glucosidases from the psychrotolerant bacterium Shewanella sp. G5 isolated from a subAntarctic ecosystem. Enzyme and Microbial Technology 2009, 45(6): 498-506.

[121] Christakopoulos P, Goodenough PW, Kekos D, Macris BJ, Claeyssens M, Bhat MK. Purification and Characterisation of an Extracellular $\beta$-Glucosidase with Transglycosylation and Exo-glucosidase Activities from Fusarium oxysporum. European Journal of Biochemistry 1994, 224(2): 379-385.

[122] Riou C, Salmon J-M, Vallier M-J, Günata Z, Barre P. Purification, characterization, and substrate specificity of a novel highly glucose-tolerant $\beta$-glucosidase fromAspergillus oryzae. Applied and Environmental Microbiology 1998, 64(10): 3607-3614.

[123] Spiridonov NA, Wilson DB. Cloning and biochemical characterization of $\mathrm{BglC}$, a $\beta$-glucosidase from the cellulolytic actinomycete Thermobifida fusca. Current Microbiology 2001, 42(4): 295-301.

[124] Lau ATY, Wong WKR. Purification and Characterization of a Major Secretory Cellobiase, Cba2, from Cellulomonas biazotea. Protein Expression and Purification 2001, 23(1): 159-166.

[125] Kudo K, Watanabe A, Ujiie S, Shintani T, Gomi K. Purification and enzymatic characterization of secretory glycoside hydrolase family 3 (GH3) aryl $\beta$-glucosidases screened from Aspergillus oryzae genome. Journal of Bioscience and Bioengineering 2015, 120(6): 614-623.

[126] Khairudin NBA, Mazlan NSF. Molecular Docking Study of BetaGlucosidase with Cellobiose, Cellotetraose and Cellotetriose. Bioinformation 2013, 9(16): 813.

[127] Rajoka MI, Idrees S, Ashfaq UA, Ehsan B, Haq A. Determination of Substrate Specificities Against $\beta$-Glucosidase A (BglA) from Thermotoga maritime: A Molecular Docking Approach. Journal of Microbiology and Biotechnology 2015, 25(1): 54-59.

[128] Rani V, Mohanram S, Tiwari R, Nain L, Arora A. Betaglucosidase: Key enzyme in determining efficiency of cellulase and biomass hydrolysis. Journal of Bioprocessing \& Biotechniques 2015, 2015.

[129] Sengupta S, Ghosh AK, Sengupta S. Purification and characterisation of a $\beta$-glucosidase (cellobiase) from a mushroom Termitomyces clypeatus. Biochimica et Biophysica Acta (BBA)Protein Structure and Molecular Enzymology 1991, 1076(2): 215220.

[130] Santos F, Garcia NFL, da Paz MF, Fonseca GG, Leite RSR. Production and characterization of $\beta$-glucosidase from Gongronella butleri by solid-state fermentation. African Journal of Biotechnology 2016, 15(16): 633-641.

[131] Saha BC, Bothast RJ. Production, purification, and characterization of a highly glucose-tolerant novel beta- 
glucosidase from Candida peltata. Applied and Environmental Microbiology 1996, 62(9): 3165-3170.

[132] Rajasree KP, Mathew GM, Pandey A, Sukumaran RK. Highly glucose tolerant $\beta$-glucosidase from Aspergillus unguis: NII 08123 for enhanced hydrolysis of biomass. Journal of Industrial Microbiology \& Biotechnology 2013, 40(9): 967-975.

[133] Yan T-R, Lin C-L. Purification and characterization of a glucosetolerant $\beta$-glucosidase from Aspergillus niger CCRC 31494. Bioscience, Biotechnology, and Biochemistry 1997, 61(6): 965-970.

[134] Ramani G, Meera B, Vanitha C, Rajendhran J, Gunasekaran P. Molecular cloning and expression of thermostable glucose-tolerant $\beta$-glucosidase of Penicillium funiculosum NCL1 in Pichia pastoris and its characterization. Journal of Industrial Microbiology \& Biotechnology 2015, 42(4): 553-565.

[135] Wright RM, Yablonsky MD, Shalita ZP, Goyal A, Eveleigh D. Cloning, characterization, and nucleotide sequence of a gene encoding Microbispora bispora BglB, a thermostable beta-glucosidase expressed in Escherichia coli. Applied and Environmental Microbiology 1992, 58(11): 3455-3465.

[136] Yang F, Yang X, Li Z, Du C, Wang J, Li S. Overexpression and characterization of a glucose-tolerant $\beta$-glucosidase from $\mathrm{T}$. aotearoense with high specific activity for cellobiose. Applied Mcrobiology and Biotechnology 2015, 99(21): 8903-8915.

[137] Jabbour D, Klippel B, Antranikian G. A novel thermostable and glucose-tolerant $\beta$-glucosidase from Fervidobacterium islandicum. Applied Microbiology and Biotechnology 2012, 93(5): 1947-1956.

[138] Zhao L, Xie J, Zhang X, Cao F, Pei J. Overexpression and characterization of a glucose-tolerant $\beta$-glucosidase from Thermotoga thermarum DSM 5069T with high catalytic efficiency of ginsenoside Rb1 to Rd. Journal of Molecular Catalysis B: Enzymatic 2013, 95: 62-69.

[139] Fang Z, Fang W, Liu J, Hong Y, Peng H, Zhang X, Sun B, Xiao Y. Cloning and characterization of a $\beta$-glucosidase from marine microbial metagenome with excellent glucose tolerance. Journal of Microbiology and Biotechnology 2010, 20(9): 1351-1358.

[140] Li G, Jiang Y, Fan X-j, Liu Y-h: Molecular cloning and characterization of a novel $\beta$-glucosidase with high hydrolyzing ability for soybean isoflavone glycosides and glucose-tolerance from soil metagenomic library. Bioresource Technology 2012, 123: 15-22.

[141] Guo B, Amano Y, Nozaki K. Improvements in Glucose Sensitivity and Stability of Trichoderma reesei $\beta$-Glucosidase Using SiteDirected Mutagenesis. PloS one 2016, 11(1): e0147301.

[142] Lundemo P, Adlercreutz P, Karlsson EN. Improved transferase/hydrolase ratio through rational design of a family $1 \beta$ glucosidase from Thermotoga neapolitana. Applied and Environmental Microbiology 2013, 79(11): 3400-3405.

[143] Wang L-X, Huang W. Enzymatic transglycosylation for glycoconjugate synthesis. Current Opinion in Chemical Biology 2009, 13(5): 592-600.

[144] Zeuner B, Jers C, Mikkelsen JD, Meyer AS: Methods for improving enzymatic trans-glycosylation for synthesis of human milk oligosaccharide biomimetics. Journal of Agricultural and Food Chemistry 2014, 62(40): 9615-9631.

[145] Hays WS, VanderJagt DJ, Bose B, Serianni AS, Glew RH: Catalytic mechanism and specificity for hydrolysis and transglycosylation reactions of cytosolic $\beta$-glucosidase from guinea pig liver. Journal of Biological Chemistry 1998, 273(52): 34941-34948.

[146] Li YK, Chir J, Chen FY. Catalytic mechanism of a family 3 betaglucosidase and mutagenesis study on residue Asp-247. Biochemical Journal 2001, 355(Pt 3): 835-840.

[147] Parry NJ, Beever, DE, Emyr O, Vandenberghe I, Van Beeumen J. Biochemical characterization and mechanism of action of a thermostable $\beta$-glucosidase purified from Thermoascus aurantiacus. Biochemical Journal 2001, 353(1): 117-127.

[148] Kaur J, Chadha BS, Kumar BA, Kaur G, Saini HS. Purification and characterization of ß-glucosidase from Melanocarpus sp. MTCC 3922. Electronic Journal of Biotechnology 2007, 10(2): 260-270.

[149] Pal S, Banik SP, Ghorai S, Chowdhury S, Khowala S. Purification and characterization of a thermostable intra-cellular $\beta$-glucosidase with transglycosylation properties from filamentous fungus Termitomyces clypeatus. Bioresource Technology 2010, 101(7): 2412-2420.

[150] Deschrevel B, Vincent JC, Ripoll C, Thellier M. Thermodynamic parameters monitoring the equilibrium shift of enzyme-catalyzed hydrolysis/synthesis reactions in favor of synthesis in mixtures of water and organic solvent. Biotechnology and Bioengineering 2003, 81(2): 167-177.

[151] Choi J-Y, Park A-R, Kim YJ, Kim J-J, Cha C-J, Yoon J-J. Purification and characterization of an extracellular beta-glucosidase produced by Phoma sp. KCTC11825BP isolated from rotten mandarin peel. Journal of Microbiology and Biotechnology 2011, 21(5): 503-508.

[152] Mai Z, Yang J, Tian X, Li J, Zhang S. Gene cloning and characterization of a novel salt-tolerant and glucose-enhanced $\beta$ glucosidase from a marine streptomycete. Applied Biochemistry and Biotechnology 2013, 169(5): 1512-1522.

[153] Krisch J, Bencsik O, Papp T, Vágvölgyi C, Takó M. Characterization of a $\beta$-glucosidase with transgalactosylation capacity from the zygomycete Rhizomucor miehei. Bioresource Technology 2012, 114: 555-560.

[154] Ng IS, Li C-W, Chan S-P, Chir J-L, Chen PT, Tong C-G, Yu S-M, Ho T-HD. High-level production of a thermoacidophilic $\beta$ glucosidase from Penicillium citrinum YS40-5 by solid-state fermentation with rice bran. Bioresource Technology 2010, 101(4) 1310-1317.

[155] Yang X, Ma R, Shi P, Huang H, Bai Y, Wang Y, Yang P, Fan Y, Yao B. Molecular characterization of a highly-active thermophilic $\beta$-glucosidase from Neosartorya fischeri P1 and its application in the hydrolysis of soybean isoflavone glycosides. PloS one 2014, 9(9): e106785.

[156] Kuo L-C, Lee K-T. Cloning, expression, and characterization of two $\beta$-glucosidases from isoflavone glycoside-hydrolyzing Bacillus subtilis natto. Journal of Agricultural and Food Chemistry 2007, 56(1): 119-125.

[157] Tajima K, Nakajima K, Yamashita H, Shiba T, Munekata M, Takai M. Cloning and sequencing of the beta-glucosidase gene from Acetobacter xylinum ATCC 23769. DNA Research 2001, 8(6): 263-269.

[158] Albaser A, Kazana E, Bennett MH, Cebeci F, Luang-In V, Spanu PD, Rossiter JT. Discovery of a Bacterial Glycoside Hydrolase Family 3 (GH3) $\beta$-Glucosidase with Myrosinase Activity from a Citrobacter Strain Isolated from Soil. Journal of Agricultural and Food Chemistry 2016, 64(7): 1520-1527.

[159] Nijikken Y, Tsukada T, Igarashi K, Samejima M, Wakagi T, Shoun H, Fushinobu S. Crystal structure of intracellular family 1 $\beta$-glucosidase BGL1A from the basidiomycete Phanerochaete chrysosporium. FEBS Letters 2007, 581(7): 1514-1520.

[160] Hernández-Guzmán A, Flores-Martínez A, Ponce-Noyola P, Villagómez-Castro JC. Purification and characterization of an extracellular $\beta$-glucosidase from Sporothrix schenckii. FEBS Open Bio 2016.

[161] Nair A, Kuwahara A, Nagase A, Yamaguchi H, Yamazaki T, Hosoya M, Omura A, Kiyomoto K, Yamaguchi M-a, Shimoyama T. Purification, gene cloning, and biochemical characterization of a $\beta$-glucosidase capable of hydrolyzing sesaminol triglucoside from Paenibacillus sp. KB0549. PloS one 2013, 8(4): e60538.

[162] Fang W, Song R, Zhang X, Zhang X, Zhang X, Wang X, Fang Z, Xiao $Y$. Characterization of a novel $\beta$-glucosidase from Gongronella sp. W5 and its application in the hydrolysis of soybean isoflavone glycosides. Journal of Agricultural and Food Chemistry 2014, 62(48): 11688-11695.

[163] Chen M, Qin Y, Liu Z, Liu K, Wang F, Qu Y. Isolation and characterization of a $\beta$-glucosidase from Penicillium decumbens and improving hydrolysis of corncob residue by using it as cellulase supplementation. Enzyme and Microbial Technology 2010, 46(6): 444-449.

[164] Dikshit R, Tallapragada P. Partial Purification and Characterization of $\beta$-glucosidase fromMonascus sanguineus. Brazilian Archives of Biology and Technology 2015, 58(2): 185191.

[165] Nazir A, Soni R, Saini H, Manhas RK, Chadha B. Regulation of expression of multiple of beta-glucosidases of Aspergillus terreus and their purification and characterization. Bioresources 2008, 4(1): $155-171$

[166] Kwon K-S, Lee J, Kang HG, Hah YC. Detection of $\beta$-glucosidase activity in polyacrylamide gels with esculin as substrate. Applied and Environmental Microbiology 1994, 60(12): 4584-4586.

[167] Qin Y, He H, Li N, Ling M, Liang Z. Isolation and characterization of a thermostable cellulase-producing Fusarium chlamydosporum. World Journal of Microbiology and Biotechnology 2010, 26(11): 1991-1997. 
[168] Dashtban M, Qin W. Overexpression of an exotic thermotolerant $\beta$-glucosidase in Trichoderma reesei and its significant increase in cellulolytic activity and saccharification of barley straw. Microbial Cell Factories 2012, 11(1): 1.

[169] Dan S, Marton I, Dekel M, Bravdo B-A, He S, Withers SG, Shoseyov O. Cloning, expression, characterization, and nucleophile identification of family 3, Aspergillus nigerß-glucosidase. Journal of Biological Chemistry 2000, 275(7): 4973-4980.

[170] Schmid G, Wandrey C. Purification and partial characterization of a cellodextrin glucohydrolase ( $\beta$-glucosidase) from Trichoderma reesei strain QM 9414. Biotechnology and Bioengineering 1987, 30(4): 571-585.

[171] Chen H, Hayn M, Esterbauer H. Purification and characterization of two extracellular $\beta$-glucosidases from Trichoderma reesei. Biochimica et Biophysica Acta (BBA)-Protein Structure and Molecular Enzymology 1992, 1121(1): 54-60.

[172] Singhania RR, Sukumaran RK, Rajasree KP, Mathew A, Gottumukkala L, Pandey A. Properties of a major $\beta$-glucosidaseBGL1 from Aspergillus niger NII-08121 expressed differentially in response to carbon sources. Process Biochemistry 2011, 46(7): 1521-1524.

[173] Sonia K, Chadha B, Badhan A, Saini H, Bhat M. Identification of glucose tolerant acid active $\beta$-glucosidases from thermophilic and thermotolerant fungi. World Journal of Microbiology and Biotechnology 2008, 24(5): 599-604.

[174] Decker C, Visser J, Schreier P. $\beta$-Glucosidase multiplicity from Aspergillus tubingensis CBS 643.92: purification and characterization of four $\beta$-glucosidases and their differentiation with respect to substrate specificity, glucose inhibition and acid tolerance. Applied Microbiology and Biotechnology 2001, 55(2): 157-163.

[175] Ramani G, Meera B, Vanitha C, Rao M, Gunasekaran P. Production, purification, and characterization of a $\beta$-glucosidase of Penicillium funiculosum NCL1. Applied Biochemistry and Biotechnology 2012, 167(5): 959-972.

[176] Verma A, Saini S, Nishad S, Kumar V, Singh S, Dubey A: Production, purification and characterization of beta-glucosidase from Bacillus subtilis strain PS isolated from sugarcane bagasse. Journal of Pure and Applied Microbiology 2013, 7(1): 803-810.

[177] Ling H, Ge J, Ping W, Xu X. Fermentation optimization by response surface methodology for enhanced production of beta-glucosidase of Aspergillus niger HDF05. Chinese Journal of Biotechnology 2011, 27(3): 419-426.

[178] Adrio JL, Demain AL. Genetic improvement of processes yielding microbial products. FEMS Microbiology Reviews 2006, 30(2): 187-214.

[179] Cowan D. Industrial enzyme technology. Trends in Biotechnology 1996, 14(6): 177-178.

[180] Chen P, Fu X, Ng TB, Ye X-Y. Expression of a secretory $\beta$ glucosidase from Trichoderma reesei in Pichia pastoris and its characterization. Biotechnology Letters 2011, 33(12): 2475-2479.

[181] Mushtaq A, Jamill A. Cloning of a $\beta$-glucosidase gene from thermophilic fungus Cheatomium thermophilum. Pakistan Journal Life and Social Science 2012, 10: 98-101.

[182] Njokweni A, Rose S, Van Zyl W. Fungal $\beta$-glucosidase expression in Saccharomyces cerevisiae. Journal of Industrial Microbiology \& Biotechnology 2012, 39(10): 1445-1452.

[183] Krogh KB, Harris PV, Olsen CL, Johansen KS, Hojer-Pedersen J, Borjesson J, Olsson L. Characterization and kinetic analysis of a thermostable GH3 $\beta$-glucosidase from Penicillium brasilianum. Applied Microbiology and Biotechnology 2010, 86(1): 143-154.

[184] Dashtban M, Qin W. Overexpression of an exotic thermotolerant beta-glucosidase in trichoderma reesei and its significant increase in cellulolytic activity and saccharification of barley straw. Microbial Cell Factories 2012, 11: 63.

[185] Jun H, Kieselbach T, Jönsson LJ. Enzyme production by filamentous fungi: analysis of the secretome of Trichoderma reesei grown on unconventional carbon source. Microbial Cell Factories 2011, 10: 68-68.

[186] Wang L, Ridgway D, Gu T, Moo-Young M. Bioprocessing strategies to improve heterologous protein production in filamentous fungal fermentations. Biotechnology Advances 2005, 23(2): 115-129.

[187] Murray P, Aro N, Collins C, Grassick A, Penttilä M, Saloheimo M, Tuohy M. Expression in Trichoderma reesei and characterisation of a thermostable family $3 \beta$-glucosidase from the moderately thermophilic fungus Talaromyces emersonii. Protein Expression and Purification 2004, 38(2): 248-257.

[188] Macauley-Patrick S, Fazenda ML, McNeil B, Harvey LM. Heterologous protein production using the Pichia pastoris expression system. Yeast 2005, 22(4): 249-270.

[189] Ahmad M, Hirz M, Pichler H, Schwab H. Protein expression in Pichia pastoris: recent achievements and perspectives for heterologous protein production. Applied Microbiology and Biotechnology 2014, 98(12): 5301-5317.

[190] Rahman Z, Shida Y, Furukawa T, Suzuki Y, Okada H, Ogasawara W, Morikawa Y. Application of Trichoderma reesei cellulase and xylanase promoters through homologous recombination for enhanced production of extracellular $\beta$-glucosidase I. Bioscience, Biotechnology, and Biochemistry 2009, 73(5): 1083-1089.

[191] Nakazawa H, Kawai T, Ida N, Shida Y, Kobayashi Y, Okada H, Tani S, Sumitani Ji, Kawaguchi T, Morikawa Y. Construction of a recombinant Trichoderma reesei strain expressing Aspergillus aculeatus $\beta$-glucosidase 1 for efficient biomass conversion. Biotechnology and Bioengineering 2012, 109(1): 92-99.

[192] Rahman Z, Shida Y, Furukawa T, Suzuki Y, Okada H, Ogasawara W, Morikawa Y. Evaluation and characterization of Trichoderma reesei cellulase and xylanase promoters. Applied Microbiology and Biotechnology 2009, 82(5): 899-908.

[193] Weld RJ, Plummer KM, Carpenter MA, Ridgway HJ. Approaches to functional genomics in filamentous fungi. Cell Research 2006 16(1): 31-44.

[194] Rosano GL, Ceccarelli EA. Recombinant protein expression in Escherichia coli: advances and challenges. Frontiers in Microbiology 2014, 5: 172.

[195] Cronan JE. Escherichia coli as an Experimental Organism. In: eLS John Wiley \& Sons, Ltd; 2001.

[196] Khow O, Suntrarachun S. Strategies for production of active eukaryotic proteins in bacterial expression system. Asian Pacific Journal of Tropical Biomedicine 2012, 2(2): 159-162.

[197] Demain AL, Vaishnav P. Production of recombinant proteins by microbes and higher organisms. Biotechnology Advances 2009, 27(3): 297-306.

[198] De Marco A. Strategies for successful recombinant expression of disulfide bond-dependent proteins in Escherichia coli. Microbial Cell Factories 2009, 8(1): 1

[199] Chang J, Park I-H, Lee Y-S, Ahn S-C, Zhou Y, Choi Y-L. Cloning, expression, and characterization of $\beta$-glucosidase from Exiguobacterium sp. DAU5 and transglycosylation activity. Biotechnology and Bioprocess Engineering 2011, 16(1): 97-106.

[200] Zahoor S, Javed M, Aftab M. Cloning and expression of $\beta$ glucosidase gene from Bacillus licheniformis into E. coli BL 21 (DE3). Biologia 2011, 66(2): 213-220.

[201] Koffi YG, Konan HK, Kouadio J, Dabonné S, Dué EA, Kouamé LP. Purification and biochemical characterization of betaglucosidase from cockroach, Periplaneta americana. Journal of Animal and Plant Science 2012, 13: 1747-1757.

[202] Qin Y, Zhang Y, He H, Zhu J, Chen G, Li W, Liang Z. Screening and Identification of a Fungal $\beta$-Glucosidase and the Enzymatic Synthesis of Gentiooligosaccharide. Applied Biochemistry and Biotechnology 2011, 163(8): 1012-1019.

[203] Michlmayr H, Schümann C, Wurbs P, Barreira Braz da Silva NM, Rogl V, Kulbe KD, del Hierro AM. A $\beta$-glucosidase from Oenococcus oeni ATCC BAA-1163 with potential for aroma release in wine: Cloning and expression in E. coli. World Journal of Microbiology \& Biotechnology 2010, 26(7): 1281-1289.

[204] Pogorzelski E, Wilkowska A. Flavour enhancement through the enzymatic hydrolysis of glycosidic aroma precursors in juices and wine beverages: a review. Flavour and Fragrance Journal 2007, 22(4): 251-254.

[205] Gueguen Y, Chemardin P, Janbon G, Arnaud A, Galzy P. A very efficient $\beta$-glucosidase catalyst for the hydrolysis of flavor precursors of wines and fruit juices. Journal of Agricultural and Food Chemistry 1996, 44(8): 2336-2340.

[206] Song X, Xue Y, Wang Q, Wu X. Comparison of three thermostable $\beta$-glucosidases for application in the hydrolysis of soybean isoflavone glycosides. Journal of Agricultural and Food Chemistry 2011, 59(5): 1954-1961.

[207] Day AJ, DuPont MS, Ridley S, Rhodes M, Rhodes MJ, Morgan MR, Williamson G. Deglycosylation of flavonoid and isoflavonoid glycosides by human small intestine and liver $\beta$-glucosidase activity. FEBS Letters 1998, 436(1): 71-75. 
[208] Donkor ON, Shah NP. Production of $\beta$-Glucosidase and Hydrolysis of Isoflavone Phytoestrogens by Lactobacillus acidophilus, Bifidobacterium lactis, and Lactobacillus casei in Soymilk. Journal of Food Science 2008, 73(1): M15-M20.

[209] Suetsugi M, Su L, Karlsberg K, Yuan Y-C, Chen S. Flavone and Isoflavone Phytoestrogens Are Agonists of Estrogen-Related Receptors11National Institutes of Health Grants ES08258 and CA44735. Molecular Cancer Research 2003, 1(13): 981-991.

[210] Belboukhari M, Cheriti A, Belboukhari N. Structure-Antioxidant Activity Relationship of Some Flavonoids Isolated from Warionia saharae (Asteraceae). Asian Journal of Chemistry 2013, 25(9): 4723-4725.

[211] Kumar S, Pandey AK. Chemistry and biological activities of flavonoids: an overview. The Scientific World Journal 2013, 2013.

[212] Batra P, Sharma AK. Anti-cancer potential of flavonoids: recent trends and future perspectives. 3 Biotech 2013, 3(6): 439-459.

[213] Yankep E, Njamen D, Fotsing MT, Fomum ZT, Mbanya J-C, Giner RM, Recio MC, Máñez S, Ríos JL. Griffonianone D, an Isoflavone with Anti-inflammatory Activity from the Root Bark of Millettia griffoniana 1. Journal of Natural Products 2003, 66(9): 1288-1290.

[214] Thilakarathna SH, Rupasinghe HPV. Flavonoid Bioavailability and Attempts for Bioavailability Enhancement. Nutrients 2013, 5(9): 3367-3387.

[215] Shin K-C, Nam H-K, Oh D-K. Hydrolysis of flavanone glycosides by $\beta$-glucosidase from Pyrococcus furiosus and its application to the production of flavanone aglycones from citrus extracts. Journal of Agricultural and Food Chemistry 2013, 61(47): 1153211540 .

[216] Schmidt S, Rainieri S, Witte S, Matern U, Martens S. Identification of a Saccharomyces cerevisiae glucosidase that hydrolyzes flavonoid glucosides. Applied and Environmental Microbiology 2011, 77(5): 1751-1757.

[217] White WLB, Arias-Garzon DI, McMahon JM, Sayre RT. Cyanogenesis in Cassava : The Role of Hydroxynitrile Lyase in Root Cyanide Production. Plant Physiology 1998, 116(4): 1219-1225.

[218] Nzwalo H, Cliff J. Konzo: From Poverty, Cassava, and Cyanogen Intake to Toxico-Nutritional Neurological Disease. PLoS Neglected Tropical Diseases 2011, 5(6): e1051.

[219] Maduagwu EN. Differential effects on the cyanogenic glycoside content of fermenting cassava root pulp by $\beta$-glucosidase and microbial activities. Toxicology Letters 1983, 15(4): 335-339.

[220] Elliston A, Collins SRA, Wilson DR, Roberts IN, Waldron KW. High concentrations of cellulosic ethanol achieved by fed batch semi simultaneous saccharification and fermentation of wastepaper. Bioresource Technology 2013, 134: 117-126.

[221] Rosales-Calderon O, Trajano HL, Duff SJB. Stability of commercial glucanase and $\beta$-glucosidase preparations under hydrolysis conditions. PeerJ 2014, 2: e402.

[222] Rather MY, Mishra S. $\beta$-Glycosidases: An alternative enzyme based method for synthesis of alkyl-glycosides. Sustainable Chemical Processes 2013, 1(1): 1.

[223] Niittynen L, Kajander K, Korpela R. Galacto-oligosaccharides and bowel function. Scandinavian Journal of Food \& Nutrition 2007, 51(2): 62.

[224] Intanon M, Arreola SL, Pham NH, Kneifel W, Haltrich D, Nguyen T-H. Nature and biosynthesis of galacto-oligosaccharides related to oligosaccharides in human breast milk. FEMS Microbiology Letters 2014, 353(2): 89-97.

[225] Cai J-W, Lu Y-D, Ben X-M. Effects of infant formula containing galacto-oligosaccharides on the intestinal microflora in infants. Chinese Journal of Contemporary Pediatrics 2008, 10(5): 629632.

[226] Torres DPM, Gonçalves MdPF, Teixeira JA, Rodrigues LR. Galacto-Oligosaccharides: Production, Properties, Applications, and Significance as Prebiotics. Comprehensive Reviews in Food Science and Food Safety 2010, 9(5): 438-454.

[227] Nagayoshi E, Furuta H, Fujii K, Takii Y. Characterization of $\beta$-glucosidase produced from Aspergillus awamori MIBA335. Journal of Biological Macromolecule 2011, 3(11): 69-82.

[228] Gao L, Gao F, Zhang D, Zhang C, Wu G, Chen S. Purification and characterization of a new $\beta$-glucosidase from Penicillium piceum and its application in enzymatic degradation of delignified corn stover. Bioresource Technology 2013, 147: 658-661.
[229] Ng I-S, Li C-W, Chan S-P, Chir J-L, Chen PT, Tong C-G, Yu S-M Ho T-HD. High-level production of a thermoacidophilic $\beta$-glucosidase from Penicillium citrinum YS40-5 by solid-state fermentation with rice bran. Bioresource Technology 2010, 101(4): 1310-1317.

[230] Yan Q, Zhou X-W, Zhou W, Li X-W, Feng M-Q, Zhou P. Purification and properties of a novel beta-glucosidase, hydrolyzing ginsenoside Rb1 to CK, from Paecilomyces Bainier. Journal of Microbiology and Biotechnology 2008, 18(6): 10811089.

[231] El-Ahmady El-Naggar N, Haroun S, Abd ElRazak A, A Owis E, Sherief A. Purification and Characterization of $\beta$-Glucosidase Produced by Aspergillus terreus Under Solid State Fermentation. Current Biotechnology 2015, 4(3): 380-386.

[232] Ašić A, Bešić L, Muhović I, Dogan S, Turan Y. Purification and Characterization of $\beta$-Glucosidase from Agaricus bisporus (White Button Mushroom). The Protein Journal 2015, 34(6): 453-461.

[233] Muensean K, Kim S. Purification and characterization of $\beta$ glucosidase produced by Trichoderma citrinoviride cultivated on microalga Chlorella vulgaris. Applied Biochemistry and Microbiology 2015, 51(1): 102-107.

[234] Saibi W, Amouri B, Gargouri A. Purification and biochemical characterization of a transglucosilating $\beta$-glucosidase of Stachybotrys strain. Applied Microbiology and Biotechnology 2007, 77(2): 293-300.

[235] Su J-H, Xu J-H, Yu H-L, He Y-C, Lu W-Y, Lin G-Q. Properties of a novel $\beta$-glucosidase from Fusarium proliferatum ECU2042 that converts ginsenoside $\mathrm{Rg} 3$ into Rh 2. Journal of Molecular Catalysis B: Enzymatic 2009, 57(1): 278-283.

[236] Nascimento CV, Souza FHM, Masui DC, Leone FA, Peralta RM, Jorge JA, Furriel RPM. Purification and biochemical properties of a glucose-stimulated $\beta$-D-glucosidase produced by Humicola grisea var. thermoidea grown on sugarcane bagasse. The Journal of Microbiology 2010, 48(1): 53-62.

[237] Anil N, Nutan M, Susan L, Digambar VG. Purification and characterization of $\beta$-glucosidase from Penicillium janthinellum mutant EU2D-21. Current Biotechnology 2017, 6: 1-8.

[238] Liu D, Zhang R, Yang X, Zhang Z, Song S, Miao Y, Shen Q. Characterization of a thermostable b-glucosidase from Aspergillus fumigatus Z5, and its functional expression in Pichia pastoris X-33. Microbial Cell Factories 2012, 11(25): 1-15.

[239] Li X, Pei J, Wu G, Shao W. Expression, Purification and Characterization of a Recombinant $\beta$-Glucosidase from Volvariella Volvacea. Biotechnology Letters 2005, 27(18): 13691373.

[240] Sørensen A, Ahring BK, Lübeck M, Ubhayasekera W, Bruno KS, Culley DE, Lübeck PS. Identifying and characterizing the most significant $\beta$-glucosidase of the novel species Aspergillus saccharolyticus. Canadian Journal of Microbiology 2012, 58(9): 1035-1046.

[241] Harnpicharnchai P, Champreda V, Sornlake W, Eurwilaichitr L. A thermotolerant $\beta$-glucosidase isolated from an endophytic fungi, Periconia sp., with a possible use for biomass conversion to sugars. Protein Expression and Purification 2009, 67(2): 61-69.

[242] Ramachandran P, Tiwari MK, Singh RK, Haw J-R, Jeya M, Lee J$\mathrm{K}$ : Cloning and characterization of a putative $\beta$-glucosidase (NfBGL595) from Neosartorya fischeri. Process Biochemistry 2012, 47(1): 99-105.

[243] Lee KW, Kim JH. Purification and characterization of betaglucosidase from Weissella cibaria 37. Journal of Microbiology and Biotechnology 2012, 22(12): 1705-1713.

[244] Wang L, Liu Q-M, Sung B-H, An D-S, Lee H-G, Kim S-G, Kim $\mathrm{S}-\mathrm{C}$, Lee S-T, Im W-T. Bioconversion of ginsenosides Rb 1, Rb 2, $\mathrm{Rc}$ and $\mathrm{Rd}$ by novel $\beta$-glucosidase hydrolyzing outer 3-O glycoside from Sphingomonas sp. 2F2: cloning, expression, and enzyme characterization. Journal of Biotechnology 2011, 156(2): 125-133.

[245] Tao Y-L, Yang D-H, Zhang Y-T, Zhang Y, Wang Z-Q, Wang Y-S Cai S-Q, Liu S-L. Cloning, expression, and characterization of the $\beta$-glucosidase hydrolyzing secoisolariciresinol diglucoside to secoisolariciresinol from Bacteroides uniformis ZL1. Applied Microbiology and Biotechnology 2014, 98(6): 2519-2531.

[246] Pei J, Pang Q, Zhao L, Fan S, Shi H: Thermoanaerobacterium thermosaccharolyticum $\beta$-glucosidase: a glucose-tolerant enzyme with high specific activity for cellobiose. Biotechnology for Biofuels 2012, 5(31): 1-10. 
[247] Breves R, Bronnenmeier K, Wild N, Lottspeich F, Staudenbauer WL, Hofemeister J. Genes encoding two different betaglucosidases of Thermoanaerobacter brockii are clustered in a common operon. Applied and Environmental Microbiology 1997, 63(10): 3902-3910.

[248] Choi IS, Wi SG, Jung SR, Patel DH, Bae H-J. Characterization and application of recombinant $\beta$-glucosidase $(\mathrm{BglH})$ from Bacillus licheniformis KCTC 1918. Journal of Wood Science 2009, 55(5): 329-334

[249] Xu H, Xiong A-S, Zhao W, Tian Y-S, Peng R-H, Chen J-M, Yao $\mathrm{Q}-\mathrm{H}$. Characterization of a glucose-, xylose-, sucrose-, and d- galactose-stimulated $\beta$-glucosidase from the alkalophilic bacterium Bacillus halodurans C-125. Current Microbiology 2011, 62(3): 833-839.

[250] Uchiyama T, Yaoi K, Miyazaki K. Glucose-tolerant $\beta$-glucosidase retrieved from a Kusaya gravy metagenome. Frontiers in Microbiology 2015, 6(548).

[251] Mai Z, Su H, Zhang S. Characterization of a Metagenome-Derived $\beta$-Glucosidase and Its Application in Conversion of Polydatin to Resveratrol. Catalysts 2016, 6(3): 35. 